\title{
RELATIVE MERITS OF COTTON AND JUTE CEMENT SACKS
}

\author{
By Robert J. Morris
}

ABSTRACT

This paper contains information concerning the tests made and the apparatus used in determining the relative merits of one type of cotton osnaburg and several types of jute burlap sacks used as containers for Portland cement. A brief description is given of the physical tests made, such as breaking strength, thread count, length, width, stretch, and, of greater importance, the resistance to failure from dropping or rough handling; also of the practical tests made, such as the service test, for which the sacks were put into actual service; the hotcement test, in which the sacks were filled with hot, freshly ground cement; the humidity test, in which samples were exposed to various atmospheric conditions; and the moisture test, during which the sacks of cement were subjected to excessively damp conditions. The results of all these tests are given in tabular and graphical form. From these data the type of sack with the most serviceability was chosen.

\section{CONTENTS}

Page

I. Introduction _......... 515

II. Methods of determining physical properties of the fabric

1. Resistance to failure from dropping

2. Breaking strength $\ldots \ldots \ldots$

3. Stretch

4. Thread count

5. Dimensions $\ldots \ldots$

6. Oil content $\ldots$

III. Methods of testing the sacks

1. Service test

2. Hot-cement test

3. Moisture test

4. Humidity test $\ldots \ldots \ldots 24$

IV. Results of sack tests_._.

V. Discussion of results . _

VI. Summary

\section{INTRODUCTION}

Much of the cement used in this country is packed, shipped, and stored in cloth containers. Whenever one of these containers is badly torn by rough handling or seriously caked by exposure to moisture, there may be a loss of both the cement and the sack. 
To meet the demand of the cement industry, approximately 75,000 ,000 cotton osnaburg sacks are required each year, representing an annual outlay of about $\$ 13,000,000$. The same number of "highcount" Dundee burlap sacks can be purchased for about $\$ 10,000,000$. The problem of reducing the expense either by increasing the life of the sacks or by finding a lower-priced sack that is no less serviceable led the Portland Cement Association, in cooperation with the Bureau of Standards, to investigate the performance of containers of various types.

A committee was appointed by the Portland Cement Association to select the types of sacks to be investigated. This committee selected and sent to the Bureau of Standards five different types of sacks, namely, one cotton osnaburg, two high-count jute Dundee, and two low-count jute Dundee. The osnaburg sack is the kind now used by the cement industry, and the particular osnaburg selected for this investigation was considered to be the best of its kind available. Of the four jute sacks, the two high-count types represent the better class of jute sacks and the remaining two are of a poorer grade.

The laboratory tests described in this paper were all conducted at the Bureau of Standards by a research fellow of the Portland Cement Association.

Each of the five types investigated was given a distinguishing number, the cotton sack being designated No. 1, the two high-count jute sacks No. 2 and No. 5, and the other two jute sacks No. 3 and No. 4. In all, over 600 sacks were used in the course of the work.

Grateful acknowledgment is made to W. D. Lober, chairman of the package committee of the Portland Cement Association, and to F. R. MeGowan, formerly of the Bureau of Standards, for helpful suggestions in connection with this investigation.

\section{METHODS OF DETERMINING PHYSICAL PROPERTIES OF THE FABRIC}

As criteria of the condition of a sack before and after exposure to any particular treatment, the following physical characteristics of the fabric were studied: Resistance to failure from dropping, breaking strength, stretch, thread count, dimensions, and oil content. Except where specifically stated otherwise, the material was always allowed to remain for at least three hours in an atmosphere of 65 per cent relative humidity at $70^{\circ} \mathrm{F} .\left(21^{\circ} \mathrm{C}\right.$.) before any physical tests were made. The characteristics or properties were determined in the following manner: 


\section{RESISTANCE TO FAILURE FROM DROPPING}

The resistance to drop failure was determined by means of the apparatus shown in Figure 1. The sack was filled with 94 pounds of Portland cement and placed lengthwise along the line where the door of a closed trapdoor joined. When this trapdoor was sprung

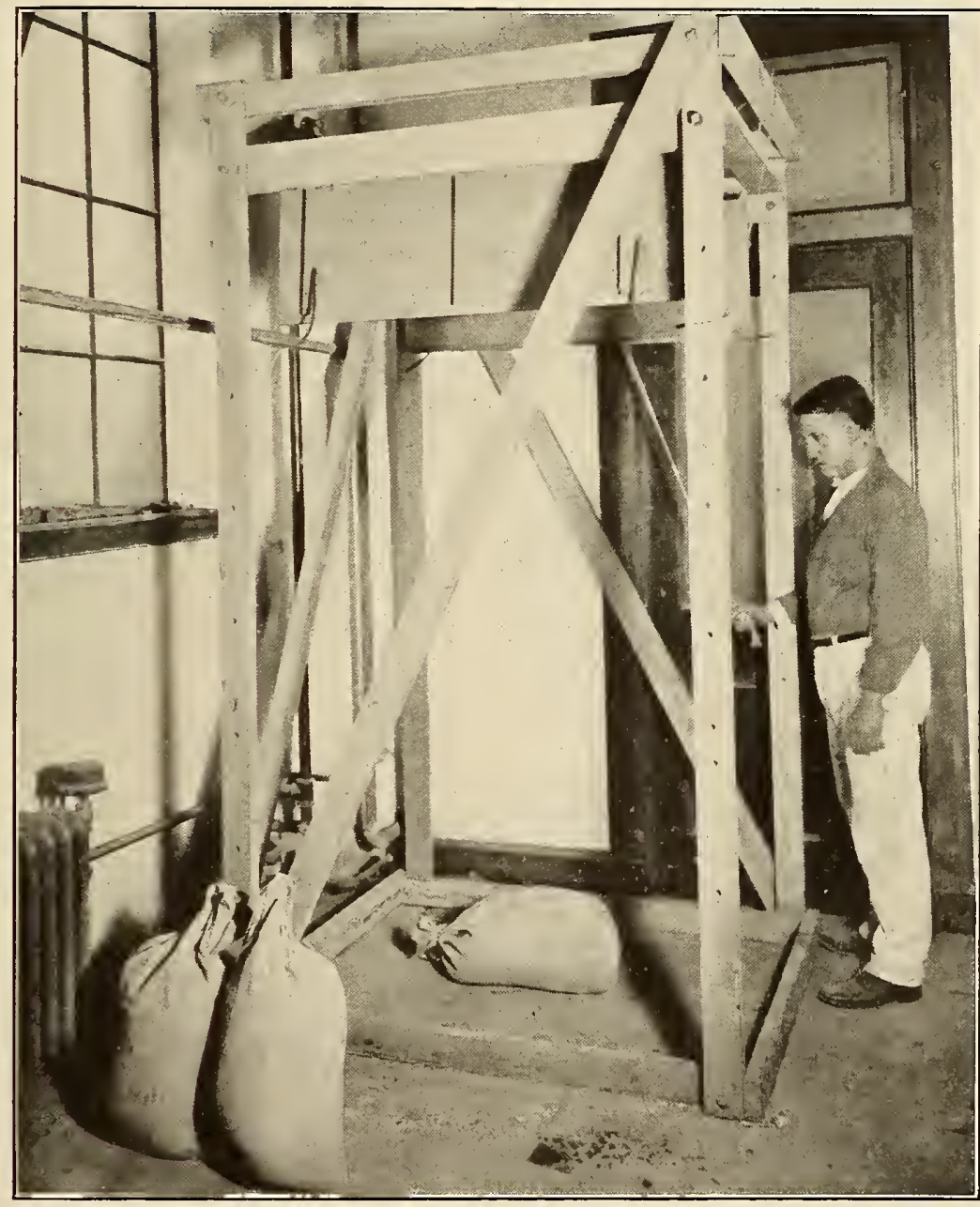

FIG. 1.-A pparatus for determining the resistance to failure from dropping

the sack was dropped a distance of 8 feet upon a smooth concrete floor. This arrangement prevented the sack from turning while falling, for no case was observed in which the sack fell on its side or 


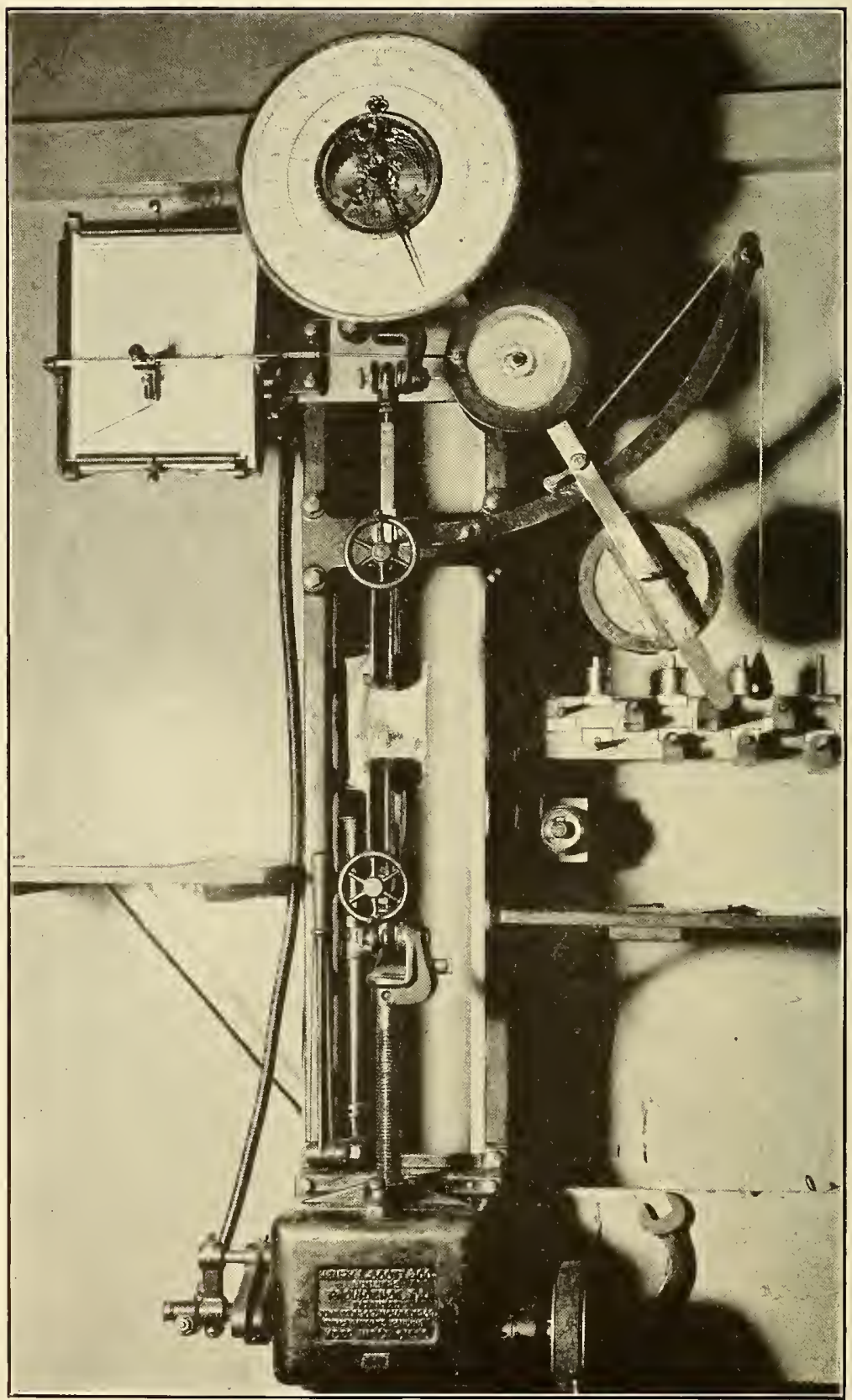

FIG. 2.-Appara'us for determining breaking s!rength 
end. The test was repeated until the fabric failed, the number of drops necessary to produce failure indicating the resistance to drop failure.

The drop test is intended to simulate the severest treatment the cement sack may receive in actual service. Preliminary work was done in order to determinc the most satisfactory method of tying the top of the sack. A number of sacks were filled and ticd very closely in such a way as to take up all possible slack at the ncck of the sack. An equal number of the same kinds of sacks were tied in the usual manner; that is, with as much space as possible between the cement and the wire used to tie the top of the sack. It was found that a fewer number of drops were required to cause failure when the sacks were tied closely, and therefore, in order to make the work progress more rapidly, the tests were made in this way. In actual service it is suggested that this information be utilized by tying the sacks near the top, so that the cement will have spacc in which to move when dropped or suddenly jarred.

\section{BREAKING STRENGTH}

The breaking strength was determined by the usual 1 by 1 by 3 inch grab method on the machine shown in Figure 2. Twenty test specimens 6 inches long by 4 inches wide were cut from each sack, 10 in the direction of the warp and 10 in the direction of the filling, care being taken that no two specimens included the same threads. No specimens for test wcre taken less than 8 inches from either selvage.

The machine used was of the inclination-balance type with a maximum capacity of 300 pounds. The lowcr or pulling jaw traveled at the uniform rate of speed of 12 inches per minute under no load. The distance between the jaws at the start of the test was 3 inches. The inside or back half of each jaw was 2 or more inches in width; the other half was 1 inch in width. The jaws used had a smooth, flat surface with the edges slightly rounded in order to prevent cutting the specimen by the jaw.

\section{STRETCH}

The stretch was detcrmined by measuring the increase in the length of the specimen at the breaking point over the length of the same specimen when under a tension of 10 pounds. Dividing this incrcasc by the original length and multiplying by 100 gives the percentagc stretch. The slight tension of 10 pounds was taken as the starting point for the stretch test, because when under no tension the length of the spccimen is indefinitc. These measurements were taken from the autographic record obtaincl during the breaking strength test describerl aborc. (Sce fig. 3.) 


\section{THREAD COUNT}

The actual number of threads in 1 inch of width was counted in each sack at three different places for both the warp and filling directions and the results averaged for each direction. These counts were made at least 6 inches apart, and no warp reading was taken at less than 8 inches from the selvage.

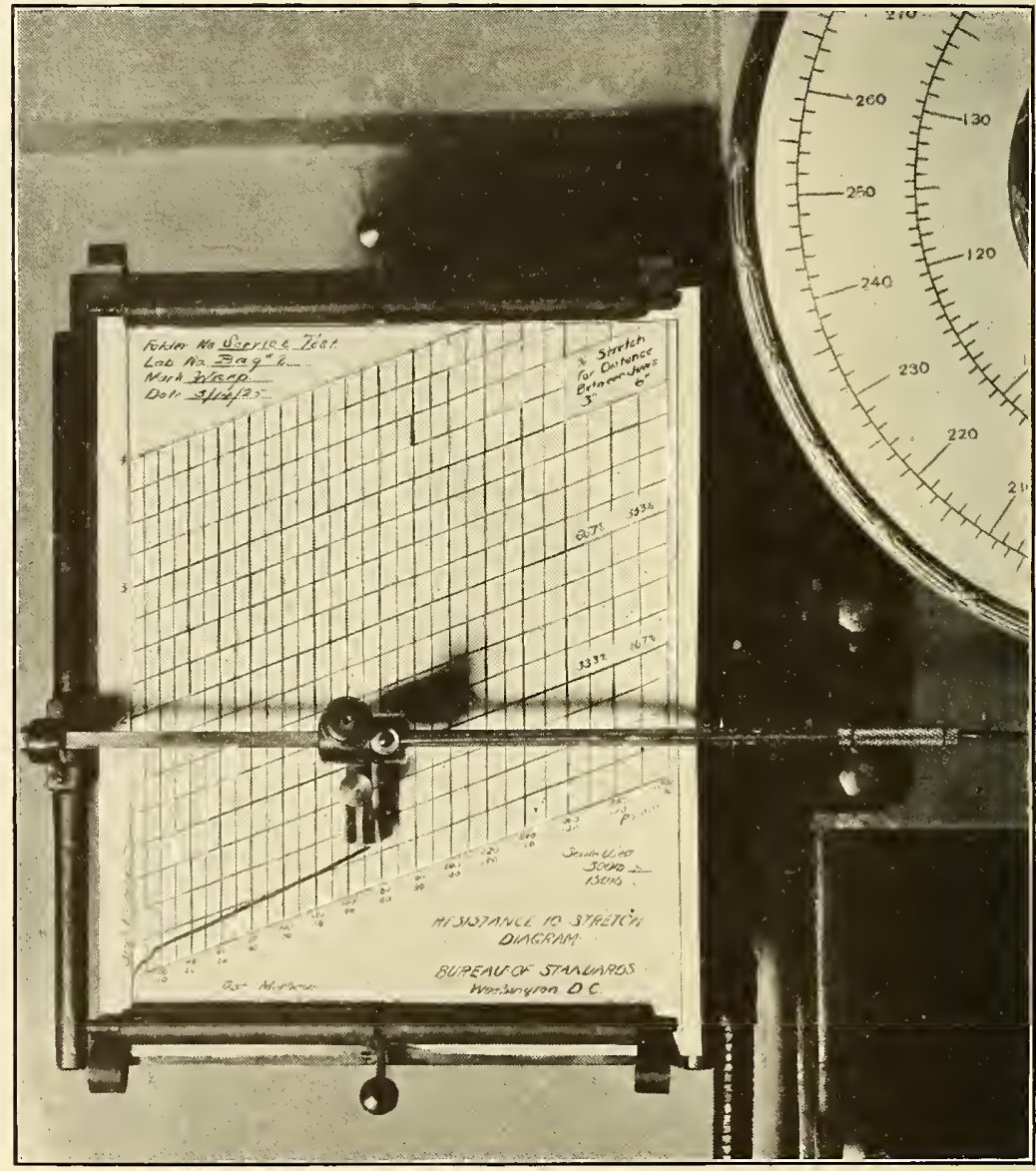

FIG. 3.-Autographic recording device for rccording stretch

\section{DIMENSIONS}

The length and width of the sack were determined by laying it on a flat surface without tension and measuring with a steel tape to the nearest one-eighth of an inch. The measurements were taken for the length and width at three different places in the sample and the results averaged. 


\section{OIL CONTENT}

In this test specimens from the cement sacks were conditioned, weighed, and extracted for six hours in a soxhlet extractor with chloroform. The apparatus was wrapped with asbestos in order to keep the chloroform hot throughout the apparatus. In case of the sacks which had been used to hold cement, the specimen was freed from the loose cement by cleaning in a vacuum. After extraction the solution was first filtered to remove dirt and any cement that had not been removed by suction. The chloroform was then cvaporated and the extract dried and weighed. This represents, of course, both the added and natural oils.

\section{METHODS OF TESTING THE SACKS}

\section{SERVICE TEST}

In the service test cement sacks were put into actual service, a certain portion being recalled at definite intervals of time and tested to determine the deterioration that had taken place. In this test 250 sacks were used, 50 of each type. They were filled with cement in the usual manner at a cement mill and shipped to a dealer in Washington, D. C. (a distance of about 200 miles), care being taken to place each test sack in the freight car at the bottom of a pile of seven. The sacks when received by the dealer were emptied and shaken for 15 minutes in a shaker customarily used for this purpose.

Five sacks of each typc were kept out for this test after each trip and the remainder sent to the mill for further service. This was done until the 250 sacks had all been recalled, the last lot having thus made 10 trips.

From every lot of 25 sacks which had made a definite number of trips from the cement mill to Washington, D. C., 10 sacks (2 of each type) were tested for resistance to drop failure, 2 sacks were used for making the breaking strength determinations, and 1 of each type was saved for future use. The stretch, thread count, oil content, and dimensions were likewise determined on all of these sacks. Values for the breaking strength were determined separately for the top and bottom portions of the sacks.

\section{HOT-CEMENT TEST}

Two tests were made to determine how the containers were affected by being filled with hot cement. In the first of these tests 75 sacks (15 of each typc) were filled at a cement mill with hot, freshly ground cement and allowed to stand for one weck. The sacks were then emptied, and 3 of cach type were sent to the Bureau of Standards for test. The rest were refilled in the same manner, and after being emptied 3 more of each type were sent to the bureau. This was repeated until the last 15 sacks had been filled five times.

$55061^{\circ}-25 \uparrow-2$ 
Of the three sacks sent to the bureau after each filling one was used for the drop test, one was tested for breaking strength, and one was kept for future reference. The dimensions, thread count, and stretch were also determined. The oil content, however, was determined only for the sacks that had been filled five times.

A second test was made with more attention to temperature control. When a sack is filled with hot cement the sack may stay hot anywhere from a few hours to sereral days, depending upon the chance the sack has to cool. Also, the temperature of the cement at the time of filling varies considerably at different mills. It is desirable, therefore, to have information on the effect of a series of temperatures maintained for extended periods of time; and a second series of tests was accordingly made consisting of holding samples cut from sacks of each type at a constant temperature in an electric oven for various periods of time. The temperatures selected ranged from 200 to $305^{\circ} \mathrm{F}$, and the time intervals during which the specimens were maintained at these temperatures ranged from 1 to $4 \mathrm{~S}$ hours. The effect of the treatment on the sacks was measured by the change produced in the breaking strength of the fabric.

\section{MOISTURE TEST}

This test is not so much a test of the deterioration of the sack as of the protection it furnished to the cement contained. It was intended to determine the effect of excessive moisture on the so-called "caking" of the cement to the sack. A long wooden frame was built in a basement room equipped with atomizers for maintaining a dense fog of moisture. A waterproof covering (such as is often used on a construction job) of rubberized cotton duck completely covered the frame. (See fig. 4.) The cement sacks under test were placed in the frame beneath the waterproof covering. The purpose of the covering was to prevent water from dripping down on the sacks from the ceiling. It did not, of course, prevent the heary fog from filling the inside of the frame. This covering thus afforded somewhat the same protection as is given to the sacks in rainy weather by the covering used by contractors.

Thirty sacks (six of each type) were weighed, then filled with fresh cement and weighed again. They were then placed under the canvas covering. The exposure continued for 18 days, 1 sack of each type being removed every third day, so that the first set of sacks was exposed 3 days, while the last set was exposed the full 18 days.

The sacks removed were first weighed to determine the moisture absorbed by the cement. They were then emptied of all the cement except the "cake," which had adhered to the sack and then weighed again. 


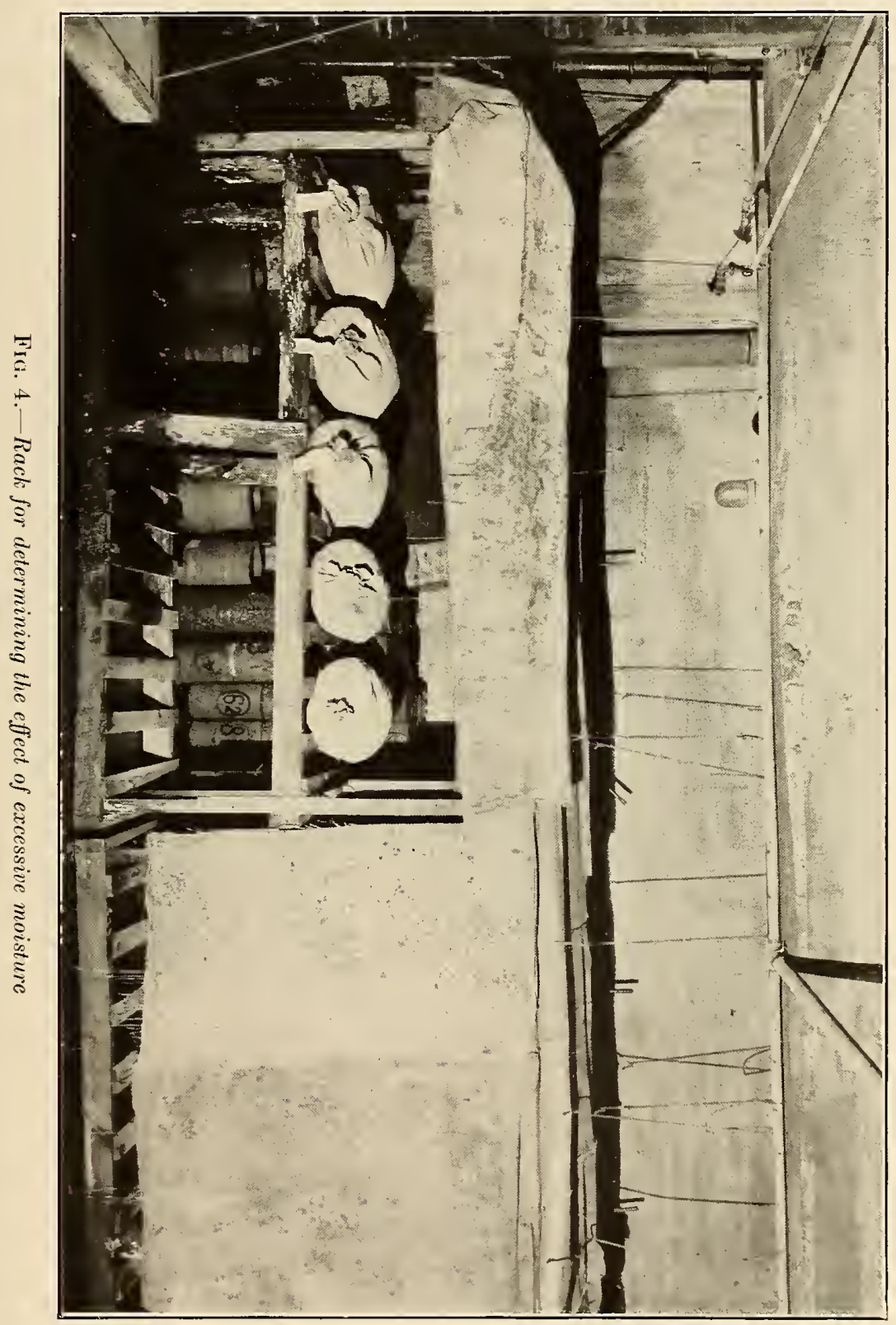




\section{HUMIDITY TEST}

In order to find the effect of humidity on the sacks themselves, samples of each type were placed in air-tight compartments. Calcium chloride was used in one of these to maintain a low humidity; in another troughs of water were used to maintain a high humidity, while a medium humidity was maintained in the third by placing the container in a conditioning room. 'The three conditions maintained in this way were, respectively, 30,90 , and 65 per cent relative humidity at a temperature of approximately $70^{\circ} \mathrm{F}$.

The changes in the breaking strength, stretch, thread count, and dimensions were used as criteria of the effect on the material.

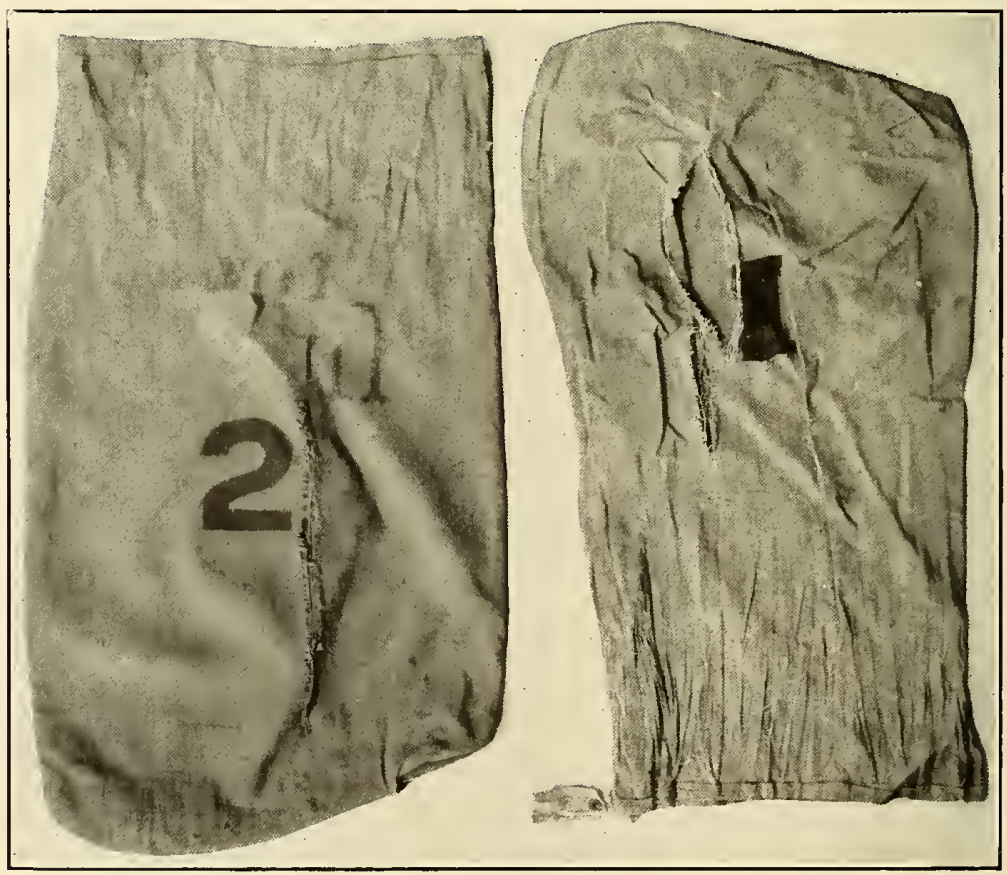

FIG. 5.-Typical result obtained of failure caused by dropping

\section{RESULTS OF SACK TESTS}

The results obtained from the measurements made on cement sacks that had been used for the actual transportation of Portland cement will be found in Table 2, and are plotted in Figure 6 . The results obtained on the hot cement, moisture, and humidity tests are given in Tables 3, 4, and 5, as well as being shown graphically in Figures 7, 8 , and 9. The loss in oil content will be found in Table 6. The results on the original sacks are shown in Table 1. 


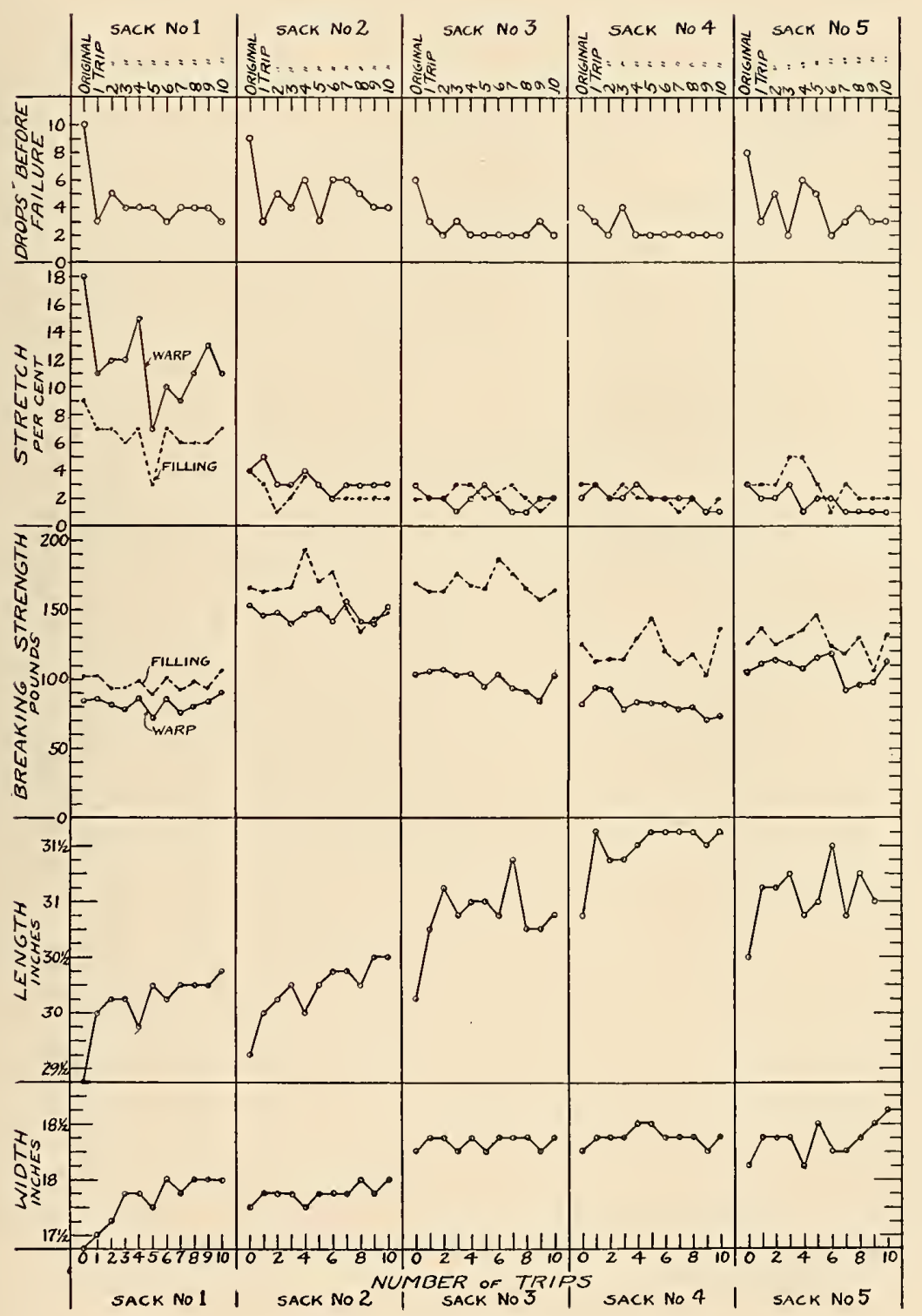

FIG. 6.-Effect of service (shipping a distance of about 200 miles) on the physical properties of the cement sacks 


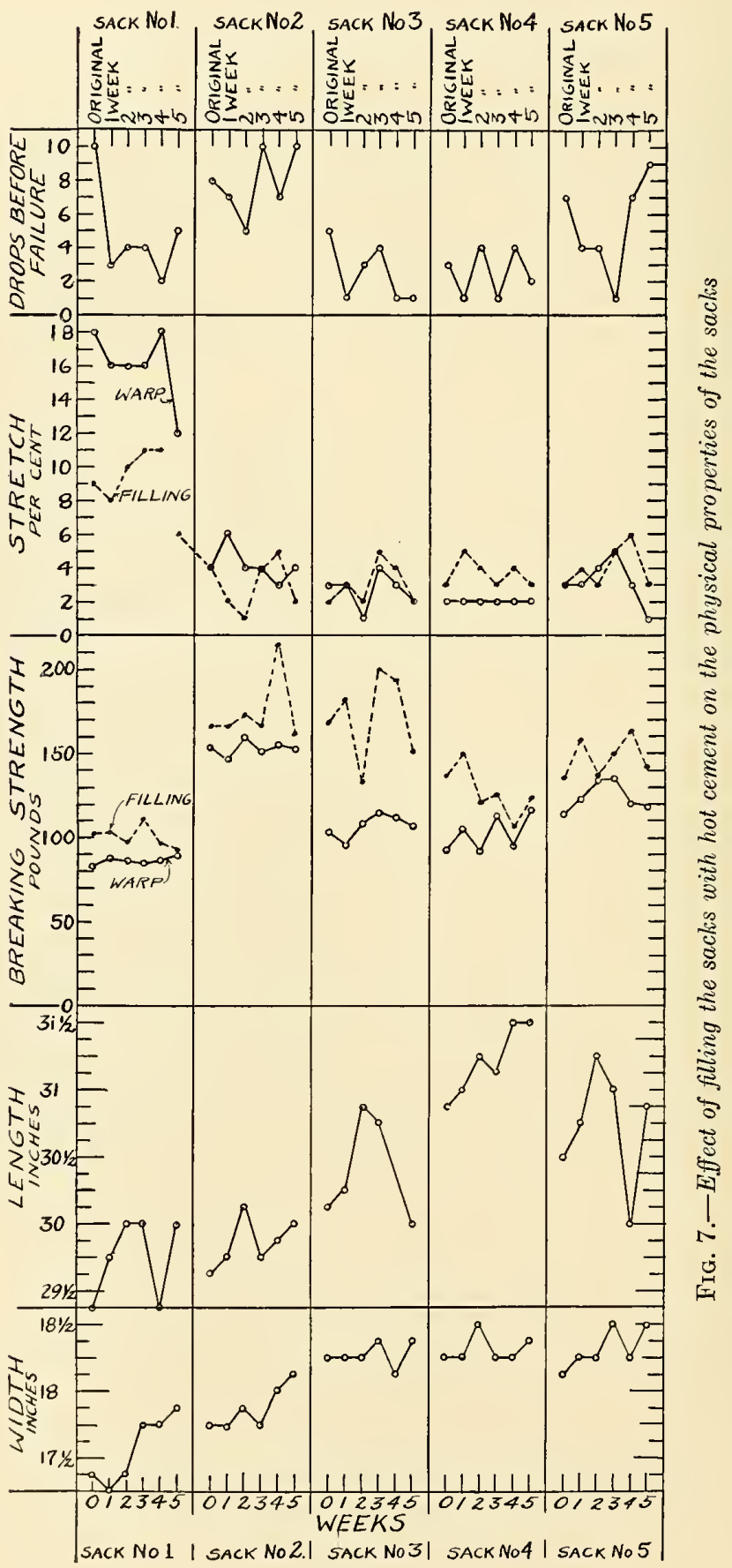




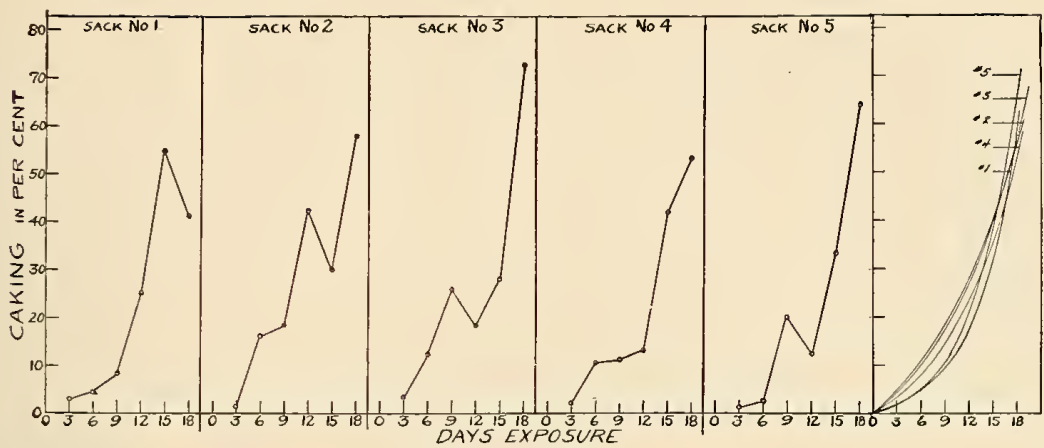

FIG. S.-Effect of exposing sacks filled with cement to a moisture atmosphere

The percentage of "caking" or adhering to the sack is shown in its relation to the number of days exposure

TABLE 1.-Physical properties of original bags

\begin{tabular}{|c|c|c|c|c|c|c|c|c|c|c|c|c|}
\hline \multirow[b]{2}{*}{ Type of sack } & \multirow{2}{*}{$\begin{array}{l}\text { Sack } \\
\text { No. }\end{array}$} & \multirow[b]{2}{*}{ Length } & \multirow[b]{2}{*}{ Width } & \multicolumn{2}{|c|}{$\begin{array}{l}\text { Threads } \\
\text { per inch }\end{array}$} & \multicolumn{2}{|c|}{$\begin{array}{c}\text { Breaking } \\
\text { strength } \\
\text { (1 by i by } \\
3 \text { inch grab) }\end{array}$} & \multirow{2}{*}{$\begin{array}{l}\text { Weight } \\
\text { per } \\
\text { square } \\
\text { yard }\end{array}$} & \multicolumn{2}{|c|}{ Stretch } & \multicolumn{2}{|c|}{ Drop test } \\
\hline & & & & Warp & $\begin{array}{l}\text { Fill- } \\
\text { ing }\end{array}$ & $W \operatorname{arp}$ & $\begin{array}{l}\text { Fill- } \\
\text { ing }\end{array}$ & & Warp & $\begin{array}{l}\text { Fill- } \\
\text { ing }\end{array}$ & $\begin{array}{l}\text { Num- } \\
\text { ber of } \\
\text { drops } \\
\text { requir- } \\
\text { ed for } \\
\text { failure }\end{array}$ & $\begin{array}{l}\text { Threads } \\
\text { broken }\end{array}$ \\
\hline $\begin{array}{l}\text { Osnaburg } \\
\text { High-count jute. } \\
\text { Low-count jute } \\
\text { Low-count jute } \\
\text { High-count jute. }\end{array}$ & $\begin{array}{l}1 \\
2 \\
3 \\
4 \\
5\end{array}$ & $\begin{array}{l}\text { Ins. } \\
293 / 8 \\
303 / 4 \\
30 \frac{7}{16} \\
307 / 8 \\
30 \frac{7}{16}\end{array}$ & $\begin{array}{l}\text { Ins. } \\
173 / 8 \\
173 / 4 \\
18 \frac{3}{16} \\
18 \frac{3}{16} \\
181 / 8\end{array}$ & $\begin{array}{l}39 \\
18 \\
121 / 2 \\
13 \\
17 \pm / 2\end{array}$ & $\begin{array}{l}30 \\
181 / 2 \\
131 / 2 \\
131 / 2 \\
20\end{array}$ & $\begin{array}{r}L b s . \\
83 \\
153 \\
103 \\
92 \\
114\end{array}$ & $\begin{array}{r}L b s . \\
102 \\
167 \\
169 \\
136 \\
137\end{array}$ & $\begin{array}{r}\text { Ozs. } \\
8.9 \\
11.8 \\
12.9 \\
11.7 \\
11.0\end{array}$ & $\begin{array}{r}P . c t \\
18 \\
4 \\
3 \\
2 \\
3\end{array}$ & $\begin{array}{r}P . c t . \\
9 \\
4 \\
2 \\
3 \\
3\end{array}$ & $\begin{array}{r}10 \\
8 \\
5 \\
3 \\
7\end{array}$ & $\begin{array}{c}\text { Warp. } \\
\text { Do. } \\
\text { Do. } \\
\text { Do. } \\
\text { Do. }\end{array}$ \\
\hline
\end{tabular}




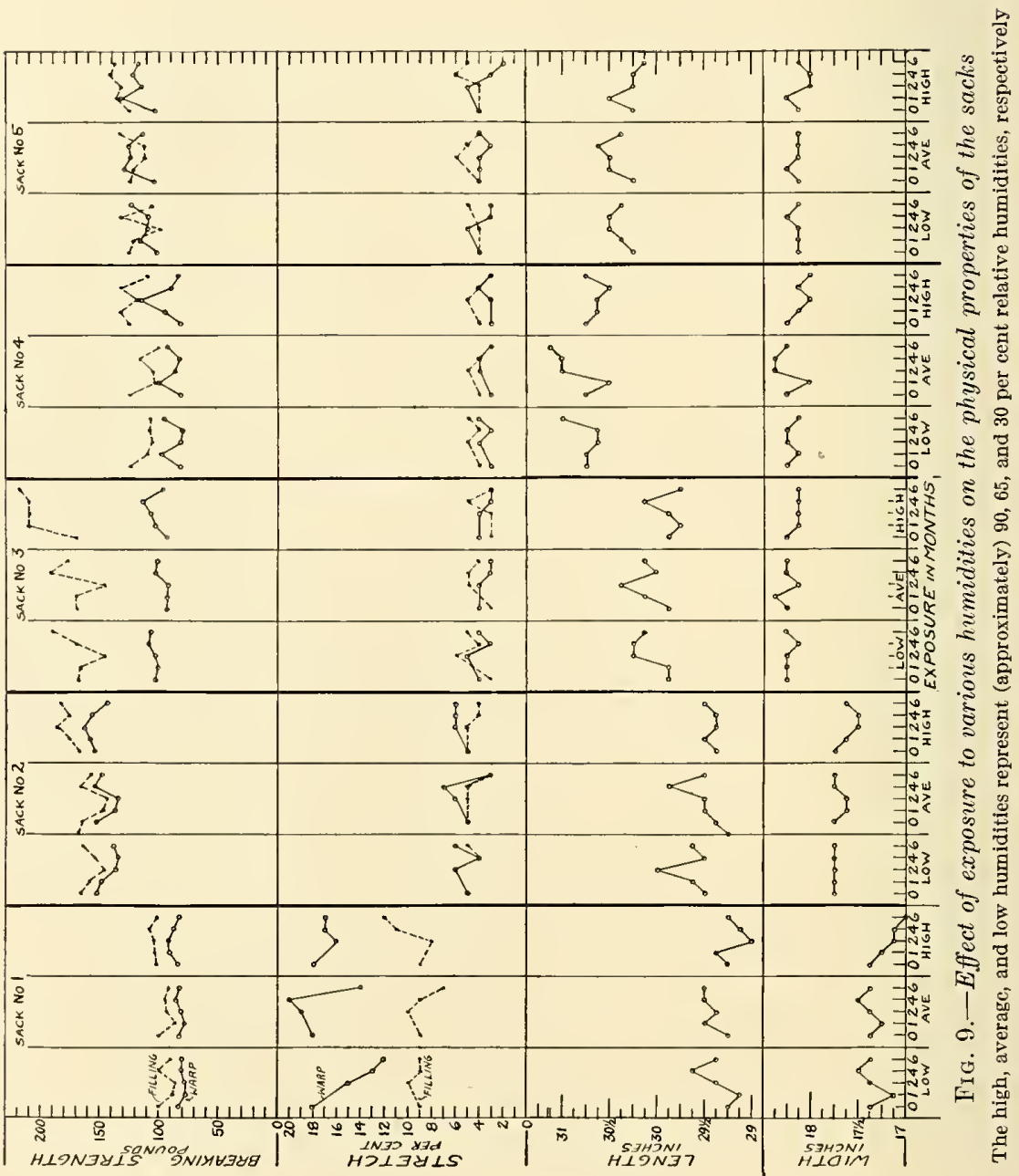




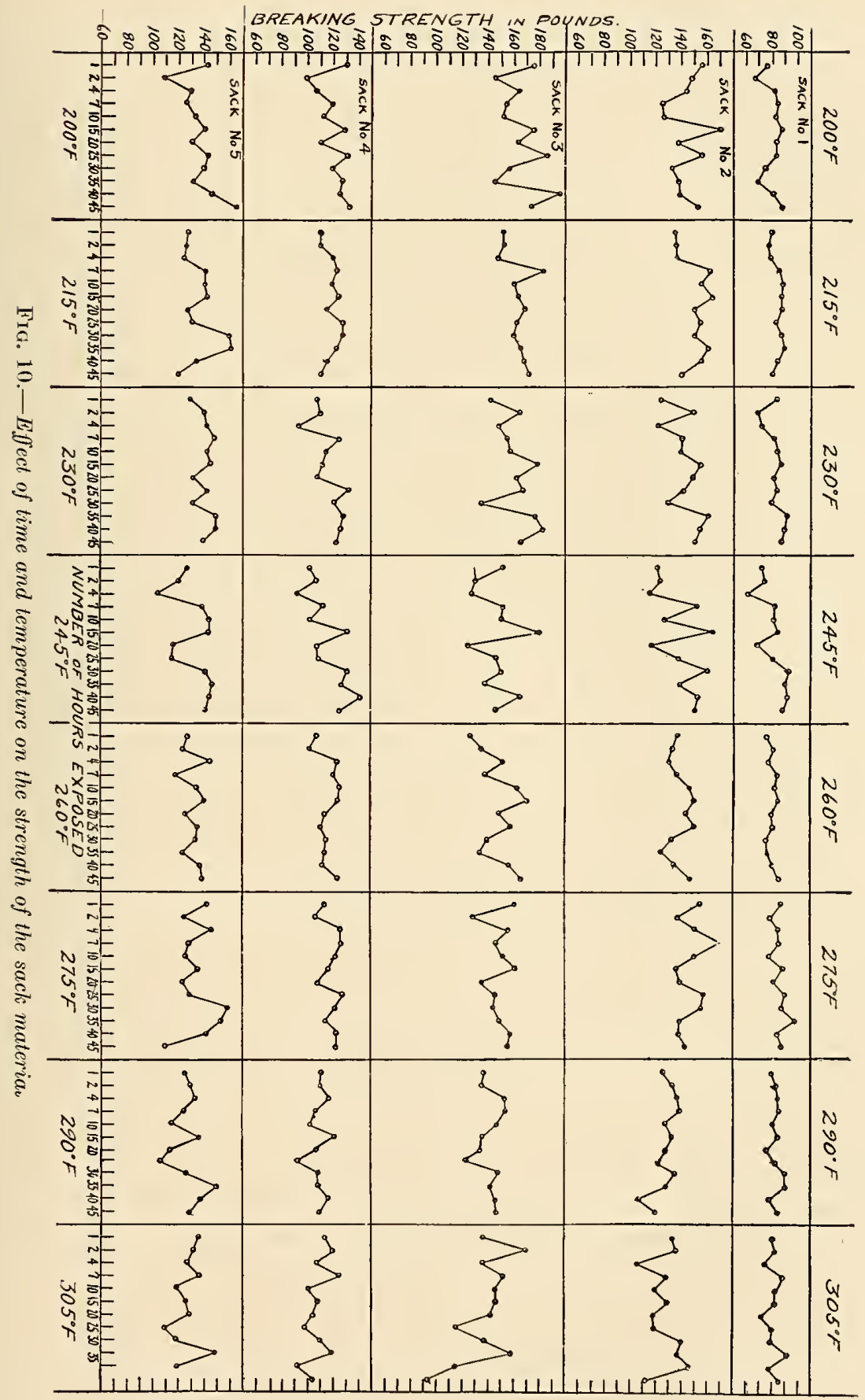


TABLE 2.-Effect of service (shipping a distance of about 200 miles) on the physical properties of the cement sacks

\begin{tabular}{|c|c|c|c|c|c|c|c|c|c|c|c|c|c|}
\hline \multirow{3}{*}{$\begin{array}{l}\text { Trip } \\
\text { No. }\end{array}$} & \multirow{3}{*}{$\begin{array}{l}\text { Sack } \\
\text { No. }\end{array}$} & \multirow{3}{*}{ Length } & \multirow{3}{*}{ Width } & \multicolumn{2}{|c|}{$\begin{array}{l}\text { Threads per } \\
\text { inch }\end{array}$} & \multicolumn{4}{|c|}{$\begin{array}{l}\text { Breaking strength } \\
\text { (1 by } 1 \text { by } 3 \text { inch grab) }\end{array}$} & \multicolumn{2}{|c|}{ Stretch } & \multicolumn{2}{|c|}{ Drop test } \\
\hline & & & & \multirow[b]{2}{*}{ Warp } & \multirow[b]{2}{*}{$\begin{array}{l}\text { Fill- } \\
\text { ing }\end{array}$} & \multicolumn{2}{|c|}{ Top } & \multicolumn{2}{|c|}{ Bottom } & \multirow[b]{2}{*}{ Warp } & \multirow[b]{2}{*}{$\begin{array}{l}\text { Fill- } \\
\text { ing }\end{array}$} & \multirow{2}{*}{$\begin{array}{l}\text { Number } \\
\text { of drops } \\
\text { required } \\
\text { for } \\
\text { failure }\end{array}$} & \multirow{2}{*}{$\begin{array}{l}\text { Threads } \\
\text { broken }\end{array}$} \\
\hline & & & & & & Warp & $\begin{array}{l}\text { Fill- } \\
\text { ing }\end{array}$ & Warp & $\begin{array}{l}\text { Fill- } \\
\text { ing }\end{array}$ & & & & \\
\hline & $\begin{array}{l}1 \\
2 \\
3 \\
4 \\
5\end{array}$ & $\begin{array}{c}\text { Ins. } \\
30 \\
30 \\
303 / 4 \\
31 \frac{9}{16} \\
31 \frac{16}{8}\end{array}$ & $\begin{array}{c}\text { Ins. } \\
171 / 2 \\
17^{3} / 4 \\
181 / 4 \\
18^{3} / 8 \\
18^{3} / 8\end{array}$ & $\begin{array}{l}381 / 2 \\
181 / 2 \\
12 \\
13 \\
171 / 2\end{array}$ & $\begin{array}{l}301 / 2 \\
181 / 2 \\
13 \\
13 \\
20\end{array}$ & $\begin{array}{r}L b s . \\
83 \\
145 \\
107 \\
103 \\
111\end{array}$ & $\begin{aligned} & L b s . \\
& 100 \\
& 157 \\
& 161 \\
& 133 \\
& 149\end{aligned}$ & $\begin{array}{r}L b s . \\
85 \\
146 \\
104 \\
102 \\
130\end{array}$ & $\begin{array}{c}\text { Lbs. } \\
105 \\
171 \\
167 \\
110 \\
144\end{array}$ & $\begin{array}{r}\text { P. ct. } \\
11 \\
5 \\
2 \\
3 \\
2\end{array}$ & $\begin{array}{r}P . c t . \\
7 \\
3 \\
2 \\
3 \\
3\end{array}$ & $\begin{array}{l}3 \\
3 \\
2 \\
2 \\
2\end{array}$ & $\begin{array}{c}\text { Warp. } \\
\text { Do. } \\
\text { Do. } \\
\text { Do. } \\
\text { Do. }\end{array}$ \\
\hline 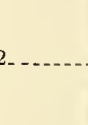 & $\begin{array}{l}1 \\
2 \\
3 \\
4 \\
5\end{array}$ & $\begin{array}{l}30 \frac{1}{16} \\
301 / 8 \\
311 / 8 \\
313 / 8 \\
311 / 8\end{array}$ & $\begin{array}{l}17 \frac{9}{16} \\
17 \frac{7}{78} \\
18 \frac{5}{16} \\
18 \frac{16}{16} \\
18 \frac{16}{16}\end{array}$ & $\begin{array}{l}39 \\
181 / 2 \\
12 \\
13 \\
17\end{array}$ & $\begin{array}{l}31 \\
181 / 2 \\
13 \\
131 / 2 \\
201 / 2\end{array}$ & $\begin{array}{r}80 \\
146 \\
105 \\
102 \\
128\end{array}$ & $\begin{array}{r}95 \\
166 \\
165 \\
126 \\
127\end{array}$ & \begin{tabular}{r|}
81 \\
148 \\
110 \\
101 \\
121
\end{tabular} & $\begin{array}{r}93 \\
163 \\
163 \\
126 \\
143\end{array}$ & \begin{tabular}{r|}
12 \\
3 \\
2 \\
2 \\
2
\end{tabular} & $\begin{array}{l}7 \\
1 \\
2 \\
2 \\
3\end{array}$ & $\begin{array}{l}5 \\
5 \\
1 \\
1 \\
4\end{array}$ & $\begin{array}{l}\text { Do. } \\
\text { Do. } \\
\text { Do. } \\
\text { Do. } \\
\text { Do. }\end{array}$ \\
\hline , & $\begin{array}{l}1 \\
2 \\
3 \\
4 \\
5\end{array}$ & $\begin{array}{l}301 / 8 \\
301 / 4 \\
307 \\
313 \\
31^{\frac{3}{16}}\end{array}$ & $\begin{array}{l}177 / 8 \\
177 / 8 \\
181 / 4 \\
18^{3} / 8 \\
18^{3} / 8\end{array}$ & $\begin{array}{l}39 \\
18 \\
12 \\
13 \\
17\end{array}$ & $\begin{array}{l}301 / 2 \\
181 / 2 \\
131 / 2 \\
121 / 2 \\
191 / 2\end{array}$ & $\begin{array}{r}79 \\
137 \\
103 \\
83 \\
122\end{array}$ & $\begin{array}{r}95 \\
155 \\
177 \\
116 \\
139\end{array}$ & $\begin{array}{r}77 \\
143 \\
103 \\
93 \\
120\end{array}$ & $\begin{array}{r}93 \\
157 \\
175 \\
131 \\
142\end{array}$ & $\begin{array}{r}12 \\
3 \\
1 \\
2 \\
3\end{array}$ & $\begin{array}{l}6 \\
2 \\
3 \\
3 \\
3 \\
5\end{array}$ & $\begin{array}{l}4 \\
4 \\
2 \\
3 \\
1\end{array}$ & $\begin{array}{l}\text { Do. } \\
\text { Do. } \\
\text { Do. } \\
\text { Do. } \\
\text { Do. }\end{array}$ \\
\hline 4 & $\begin{array}{l}1 \\
2 \\
3 \\
4 \\
5\end{array}$ & $\begin{array}{l}297 / 8 \\
30 \\
31 \\
311 / 2 \\
307 / 8\end{array}$ & $\begin{array}{l}177 / 8 \\
173 / 4 \\
18^{3} / 8 \\
181 / 2 \\
181 / 8\end{array}$ & $\begin{array}{l}38 \\
18 \\
121 / 2 \\
13 \\
171 / 2\end{array}$ & $\begin{array}{l}29 \\
18 \\
121 / 2 \\
13 \\
20\end{array}$ & $\begin{array}{r}82 \\
148 \\
105 \\
93 \\
117\end{array}$ & $\begin{array}{r}99 \\
190 \\
165 \\
138 \\
149\end{array}$ & \begin{tabular}{r|r|}
89 & 102 \\
143 & 95 \\
116 &
\end{tabular} & $\begin{array}{r}99 \\
195 \\
169 \\
143 \\
140\end{array}$ & $\begin{array}{r}15 \\
4 \\
2 \\
3 \\
1\end{array}$ & $\begin{array}{l}7 \\
4 \\
3 \\
2 \\
5\end{array}$ & $\begin{array}{l}4 \\
6 \\
1 \\
1 \\
5\end{array}$ & $\begin{array}{l}\text { Do. } \\
\text { Do. } \\
\text { Do. } \\
\text { Do. } \\
\text { Do. }\end{array}$ \\
\hline 5 & $\begin{array}{l}1 \\
2 \\
3 \\
4 \\
5\end{array}$ & $\begin{array}{l}301 / 4 \\
301 / 4 \\
31 \\
315 / 8 \\
31\end{array}$ & $\begin{array}{l}17^{3} \\
17^{7} \\
181 \\
181 \\
181 / 2 \\
18\end{array}$ & $\begin{array}{l}39 \\
18 \\
12 \\
121 / 2 \\
17\end{array}$ & $\begin{array}{l}291 / 2 \\
181 / 2 \\
13 \\
131 / 2 \\
19\end{array}$ & $\begin{array}{r}73 \\
148 \\
95 \\
97 \\
117\end{array}$ & $\begin{array}{r}90 \\
168 \\
156 \\
156 \\
153\end{array}$ & $\begin{array}{r}70 \\
162 \\
92 \\
89 \\
126\end{array}$ & $\begin{array}{r}87 \\
171 \\
172 \\
154 \\
159\end{array}$ & $\begin{array}{l}7 \\
3 \\
3 \\
2 \\
2\end{array}$ & $\begin{array}{l}3 \\
3 \\
2 \\
2 \\
3\end{array}$ & $\begin{array}{l}4 \\
3 \\
1 \\
1 \\
4\end{array}$ & $\begin{array}{c}\text { Do. } \\
\text { Sewing. } \\
\text { Warp. } \\
\text { Do. } \\
\text { Do. }\end{array}$ \\
\hline 6. & $\begin{array}{l}1 \\
2 \\
3 \\
4 \\
5\end{array}$ & $\begin{array}{l}30^{1 / 8} 8 \\
30^{3} 8 \\
30^{7} / 8 \\
3158 \\
311 / 2\end{array}$ & \begin{tabular}{l|l}
18 \\
$177 / 8$ \\
$183 / 8$ \\
183 \\
$182 / 4$
\end{tabular} & $\begin{array}{l}39 \\
18 \\
12 \\
121 / 2 \\
17\end{array}$ & $\begin{array}{l}30 \\
181 / 2 \\
13 \\
13 \\
191 / 2\end{array}$ & $\begin{array}{r}87 \\
140 \\
104 \\
91 \\
138\end{array}$ & $\begin{array}{r}96 \\
180 \\
188 \\
126 \\
128\end{array}$ & $\begin{array}{r}85 \\
141 \\
104 \\
95 \\
118\end{array}$ & $\begin{array}{l}103 \\
174 \\
185 \\
135 \\
143\end{array}$ & $\begin{array}{r}10 \\
2 \\
2 \\
2 \\
2\end{array}$ & $\begin{array}{l}7 \\
2 \\
2 \\
2 \\
1\end{array}$ & $\begin{array}{l}3 \\
6 \\
1 \\
1 \\
1\end{array}$ & $\begin{array}{l}\text { Do. } \\
\text { Do. } \\
\text { Do. } \\
\text { Do. } \\
\text { Do. }\end{array}$ \\
\hline & $\begin{array}{l}1 \\
2 \\
3 \\
4 \\
5\end{array}$ & $\begin{array}{l}301 / 4 \\
303 / 8 \\
31^{3 / 3} \\
315 \\
30 \% 8\end{array}$ & $\begin{array}{l}177 / 8 \\
177 / 8 \\
183 / 8 \\
183 / 8 \\
18 \% / 4\end{array}$ & $\begin{array}{l}38 \\
18 \\
12 \\
121 / 2 \\
17\end{array}$ & $\begin{array}{l}30 \\
18 \\
13 \\
131 / 2 \\
201 / 2\end{array}$ & $\begin{array}{r}76 \\
159 \\
93 \\
95 \\
102\end{array}$ & $\begin{array}{r}99 \\
144 \\
168 \\
120 \\
129\end{array}$ & $\begin{array}{r}75 \\
150 \\
93 \\
82 \\
100\end{array}$ & $\begin{array}{r}85 \\
148 \\
183 \\
122 \\
127\end{array}$ & $\begin{array}{l}9 \\
3 \\
2 \\
2 \\
1\end{array}$ & $\begin{array}{l}6 \\
2 \\
3 \\
1 \\
3\end{array}$ & $\begin{array}{l}4 \\
6 \\
1 \\
1 \\
2\end{array}$ & $\begin{array}{l}\text { Do. } \\
\text { Do. } \\
\text { Do. } \\
\text { Do. } \\
\text { Do. }\end{array}$ \\
\hline 8_- & $\begin{array}{l}1 \\
2 \\
3 \\
4 \\
5\end{array}$ & $\begin{array}{l}301 / 4 \\
301 / 4 \\
30^{3} \\
315 \\
311 / 4 \\
31 / 4\end{array}$ & $\begin{array}{l}18 \\
177 / 8 \\
183 / 8 \\
183 / 8 \\
183 / 8\end{array}$ & $\begin{array}{l}39 \\
18 \\
12 \\
121 / 2 \\
17\end{array}$ & $\begin{array}{l}291 / 2 \\
18 \\
13 \\
131 / 2 \\
181 / 2\end{array}$ & $\begin{array}{r}80 \\
141 \\
91 \\
89 \\
104\end{array}$ & $\begin{array}{r}99 \\
133 \\
165 \\
127 \\
140\end{array}$ & & & $\begin{array}{r}11 \\
3 \\
2 \\
2 \\
1\end{array}$ & $\begin{array}{l}6 \\
2 \\
2 \\
2 \\
2\end{array}$ & $\begin{array}{l}4 \\
5 \\
1 \\
1 \\
3\end{array}$ & $\begin{array}{c}\text { Do. } \\
\text { Sewing. } \\
\text { Warp. } \\
\text { Do. } \\
\text { Do. }\end{array}$ \\
\hline $9-$ & $\begin{array}{l}1 \\
2 \\
3 \\
4 \\
5\end{array}$ & $\begin{array}{l}301 / 8 \\
301 / 2 \\
30^{3} / 4 \\
31^{1 / 2} \\
31\end{array}$ & $\begin{array}{l}18 \\
177 / 8 \\
181 / 1 \\
181 / 4 \\
181 / 2\end{array}$ & $\begin{array}{l}39 \\
18 \\
12 \\
121 / 2 \\
171 / 2\end{array}$ & $\begin{array}{l}291 / 2 \\
18 \\
131 / 2 \\
131 / 2 \\
20\end{array}$ & $\begin{array}{r}85 \\
140 \\
84 \\
80 \\
106\end{array}$ & $\begin{array}{r}94 \\
143 \\
157 \\
113 \\
116\end{array}$ & & & $\begin{array}{r}13 \\
3 \\
2 \\
1 \\
1\end{array}$ & $\begin{array}{l}6 \\
2 \\
1 \\
1 \\
2\end{array}$ & $\begin{array}{l}4 \\
4 \\
2 \\
1 \\
2\end{array}$ & \\
\hline $10_{-}$ & $\begin{array}{l}1 \\
2 \\
3 \\
4 \\
5\end{array}$ & $\begin{array}{l}303 / 8 \\
301 / 2 \\
307 / 8 \\
315 / 8 \\
311 / 8\end{array}$ & $\begin{array}{l}18 \\
18 \\
18^{3} / 8 \\
18^{3} / 8 \\
188^{1 / 2}\end{array}$ & $\begin{array}{l}39 \\
181 / 2 \\
121 / 2 \\
121 \\
171 / 2 \\
\end{array}$ & $\begin{array}{l}29 \\
181 / 2 \\
13 \\
131 / 2 \\
201 / 2\end{array}$ & $\begin{array}{r}90 \\
152 \\
102 \\
83 \\
120\end{array}$ & $\begin{array}{l}107 \\
149 \\
163 \\
147 \\
153\end{array}$ & & - & $\begin{array}{r}11 \\
3 \\
2 \\
1 \\
1\end{array}$ & $\begin{array}{l}7 \\
2 \\
2 \\
2 \\
2\end{array}$ & $\begin{array}{l}3 \\
4 \\
1 \\
1 \\
2\end{array}$ & \\
\hline
\end{tabular}


TABLE 3.-Effect of filling with hot cement on the physical properties of the sacks

\begin{tabular}{|c|c|c|c|c|c|c|c|c|c|c|c|c|}
\hline \multirow{2}{*}{$\begin{array}{c}\text { Period } \\
\text { of } \\
\text { time } \\
\text { in } \\
\text { weeks }\end{array}$} & \multirow{2}{*}{$\begin{array}{l}\text { Sack } \\
\text { No. }\end{array}$} & \multirow{2}{*}{ Length } & \multirow{2}{*}{ Width } & \multicolumn{2}{|c|}{$\begin{array}{l}\text { Threads per } \\
\text { inch }\end{array}$} & \multicolumn{2}{|c|}{$\begin{array}{c}\text { Breaking } \\
\text { strength (1 by } \\
1 \text { by } 3 \text { inch } \\
\text { grab) }\end{array}$} & \multirow{2}{*}{$\begin{array}{l}\text { Weight } \\
\text { per } \\
\text { square } \\
\text { yard }\end{array}$} & \multicolumn{2}{|c|}{ Stretch } & \multicolumn{2}{|c|}{ Drop test } \\
\hline & & & & Warp & $\begin{array}{l}\text { Fill- } \\
\text { ing }\end{array}$ & Warp & Filling & & Warp & Filling & $\begin{array}{l}\text { Number } \\
\text { of drops } \\
\text { required } \\
\text { for } \\
\text { failure }\end{array}$ & $\begin{array}{c}\text { Threads } \\
\text { broken }\end{array}$ \\
\hline & $\begin{array}{l}1 \\
2 \\
3 \\
4 \\
5\end{array}$ & $\begin{array}{c}\text { Inches } \\
29^{3} / 4 \\
293 \\
301 \\
31 \\
30^{3} \\
4\end{array}$ & $\begin{array}{r}\text { Inches } \\
171 / 4 \\
173 / 4 \\
181 / 4 \\
181 / 4 \\
181 / 4\end{array}$ & $\begin{array}{l}39 \\
18 \\
12 \\
13 \\
17\end{array}$ & $\begin{array}{l}30 \\
18 \\
121 / 2 \\
14 \\
20\end{array}$ & $\begin{array}{r}\text { Pounds } \\
87 \\
146 \\
96 \\
105 \\
123\end{array}$ & $\begin{array}{c}\text { Pounds } \\
103 \\
166 \\
185 \\
161 \\
159\end{array}$ & $\begin{array}{r}\text { Ounces } \\
8.8 \\
13.9 \\
13.6 \\
12.3 \\
11.2\end{array}$ & $\begin{array}{r}\text { Per cent } \\
16 \\
6 \\
3 \\
2 \\
3\end{array}$ & $\begin{array}{r}\text { Per cent } \\
8 \\
2 \\
3 \\
5 \\
4\end{array}$ & $\begin{array}{l}3 \\
7 \\
1 \\
1 \\
4\end{array}$ & $\begin{array}{c}\text { Warp. } \\
\text { Do. } \\
\text { Do. } \\
\text { Do. } \\
\text { Do. }\end{array}$ \\
\hline & $\begin{array}{l}1 \\
2 \\
3 \\
4 \\
5\end{array}$ & $\begin{array}{l}30 \\
301 / 8 \\
307 / 8 \\
311 / 4 \\
311 / 4\end{array}$ & $\begin{array}{l}17^{3} / 8 \\
177 / 8 \\
181 / 4 \\
181 / 2 \\
181 / 4\end{array}$ & $\begin{array}{l}38 \\
18 \\
12 \\
121 / 2 \\
17\end{array}$ & $\begin{array}{l}29 \\
171 / 2 \\
121 / 2 \\
14 \\
19\end{array}$ & $\begin{array}{r}86 \\
159 \\
108 \\
92 \\
134\end{array}$ & $\begin{array}{r}97 \\
174 \\
133 \\
122 \\
137\end{array}$ & $\begin{array}{r}8.9 \\
12.1 \\
12.3 \\
12.0 \\
11.3\end{array}$ & $\begin{array}{r}16 \\
4 \\
1 \\
2 \\
4\end{array}$ & $\begin{array}{r}10 \\
1 \\
2 \\
4 \\
3\end{array}$ & $\begin{array}{l}4 \\
5 \\
5 \\
4 \\
4\end{array}$ & $\begin{array}{c}\text { Do. } \\
\text { Sewing } \\
\text { Walp. } \\
\text { Do. } \\
\text { Do. }\end{array}$ \\
\hline & $\begin{array}{l}1 \\
2 \\
3 \\
4 \\
5\end{array}$ & $\begin{array}{l}30 \\
29^{3} / 4 \\
30^{3} / 4 \\
31^{1 / 8} \\
31\end{array}$ & $\begin{array}{l}17^{3} / 4 \\
173 / 4 \\
183 / 8 \\
181 / 4 \\
181 / 2\end{array}$ & $\begin{array}{l}39 \\
18 \\
12 \\
121 / 2 \\
17\end{array}$ & $\begin{array}{l}311 / 2 \\
171 / 2 \\
131 / 2 \\
13 \\
19\end{array}$ & $\begin{array}{r}85 \\
151 \\
115 \\
113 \\
135\end{array}$ & $\begin{array}{l}111 \\
167 \\
200 \\
126 \\
150\end{array}$ & $\begin{array}{r}9.7 \\
12.4 \\
12.6 \\
12.8 \\
11.6\end{array}$ & $\begin{array}{r}16 \\
4 \\
4 \\
2 \\
5\end{array}$ & $\begin{array}{r}11 \\
4 \\
5 \\
3 \\
5\end{array}$ & $\begin{array}{r}4 \\
10 \\
4 \\
1 \\
1\end{array}$ & $\begin{array}{c}\text { Do. } \\
\text { Sewing. } \\
\text { Warp. } \\
\text { Do. } \\
\text { Do. }\end{array}$ \\
\hline & $\begin{array}{l}1 \\
2 \\
3 \\
4 \\
5\end{array}$ & $\begin{array}{l}293 / 8 \\
297 / 8 \\
30^{3} / 8 \\
311 / 2 \\
30\end{array}$ & $\begin{array}{l}173 / 4 \\
18 \\
181 / 8 \\
181 / 4 \\
181 / 4\end{array}$ & $\begin{array}{l}39 \\
18 \\
12 \\
12^{1} / 2 \\
17\end{array}$ & $\begin{array}{l}291 / 2 \\
171 / 2 \\
131 / 2 \\
13 \\
191 / 2\end{array}$ & $\begin{array}{r}86 \\
156 \\
112 \\
95 \\
120\end{array}$ & $\begin{array}{r}96 \\
214 \\
194 \\
106 \\
164\end{array}$ & $\begin{array}{r}8.9 \\
13.1 \\
13.1 \\
12.4 \\
11.3\end{array}$ & $\begin{array}{r}18 \\
3 \\
3 \\
2 \\
3\end{array}$ & $\begin{array}{r}11 \\
5 \\
4 \\
4 \\
6\end{array}$ & $\begin{array}{l}2 \\
7 \\
1 \\
4 \\
7\end{array}$ & $\begin{array}{l}\text { Do. } \\
\text { Do. } \\
\text { Do. } \\
\text { Do. } \\
\text { Do. }\end{array}$ \\
\hline & $\begin{array}{l}1 \\
2 \\
3 \\
4 \\
5\end{array}$ & $\begin{array}{l}30 \\
30 \\
303 / 4 \\
311 / 2 \\
307 / 8\end{array}$ & $\begin{array}{l}177 / 8 \\
1818 \\
18^{3} / 8 \\
18^{3} / 8 \\
18^{\frac{3}{16}}\end{array}$ & $\begin{array}{l}39 \\
18 \\
12 \\
13 \\
171 / 2\end{array}$ & $\begin{array}{l}291 / 2 \\
181 / 2 \\
13 \\
13 \\
191 / 2\end{array}$ & $\begin{array}{r}89 \\
152 \\
107 \\
117 \\
118\end{array}$ & $\begin{array}{r}92 \\
162 \\
151 \\
125 \\
142\end{array}$ & $\begin{array}{r}8.6 \\
11.4 \\
12.7 \\
12.3 \\
11.5\end{array}$ & $\begin{array}{r}12 \\
4 \\
2 \\
2 \\
1\end{array}$ & $\begin{array}{l}6 \\
2 \\
2 \\
3 \\
3\end{array}$ & $\begin{array}{r}5 \\
10 \\
1 \\
2 \\
9\end{array}$ & $\begin{array}{l}\text { Do. } \\
\text { Do. } \\
\text { Do. } \\
\text { Do. } \\
\text { Do. }\end{array}$ \\
\hline
\end{tabular}

TABLE 4.-Effect of exposing sacks filled with cement to a moist atmosphere

\begin{tabular}{|c|c|c|c|c|c|c|c|c|}
\hline Sack No. & $\begin{array}{l}\text { Days' } \\
\text { exposure }\end{array}$ & $\begin{array}{l}\text { Original } \\
\text { weight } \\
\text { of sack }\end{array}$ & $\begin{array}{l}\text { Original } \\
\text { weight } \\
\text { of sack } \\
\text { and } \\
\text { cement }\end{array}$ & $\begin{array}{l}\text { Weight } \\
\text { of sack } \\
\text { and } \\
\text { cement } \\
\text { after } \\
\text { exposure }\end{array}$ & $\begin{array}{l}\text { Weight } \\
\text { of sack } \\
\text { and } \\
\text { "cake" } \\
\text { after } \\
\text { exposure }\end{array}$ & $\begin{array}{c}\text { Increase } \\
\text { in weight } \\
\text { of sack } \\
\text { and } \\
\text { cement }\end{array}$ & $\begin{array}{l}\text { Weight } \\
\text { of "cake" }\end{array}$ & $\begin{array}{l}\text { Percent- } \\
\text { age of } \\
\text { cement } \\
\text { "caked" }\end{array}$ \\
\hline $\begin{array}{l}1 \\
2 \\
3 \\
5\end{array}$ & $\begin{array}{l}3 \\
3 \\
3 \\
3 \\
3\end{array}$ & $\begin{array}{r}\text { Pound } \\
0.45 \\
.64 \\
.78 \\
.71 \\
.60\end{array}$ & $\begin{array}{r}\text { Pounds } \\
92.4 \\
93.1 \\
92.4 \\
92.4 \\
92.4\end{array}$ & $\begin{array}{r}\text { Pounds } \\
93.3 \\
94.1 \\
93.2 \\
93.2 \\
93.0\end{array}$ & $\begin{array}{r}\text { Pounds } \\
3.1 \\
1.7 \\
3.8 \\
2.6 \\
2.0\end{array}$ & $\begin{array}{r}\text { Pounds } \\
0.9 \\
1.0 \\
.8 \\
.8 \\
.6\end{array}$ & $\begin{array}{r}\text { Pounds } \\
2.65 \\
1.06 \\
3.02 \\
1.89 \\
1.40\end{array}$ & $\begin{array}{r}\text { Percent } \\
2.9 \\
1.1 \\
3.3 \\
2.1 \\
1.5\end{array}$ \\
\hline $\begin{array}{l}1 \\
1_{2} \\
3 \\
4 \\
5\end{array}$ & $\begin{array}{l}6 \\
6 \\
6 \\
6 \\
6\end{array}$ & $\begin{array}{r}.44 \\
.66 \\
.74 \\
.69 \\
.62\end{array}$ & $\begin{array}{l}94.9 \\
93.8 \\
93.4 \\
93.1 \\
92.4\end{array}$ & $\begin{array}{l}96.1 \\
96.8 \\
95.6 \\
94.3 \\
93.4\end{array}$ & $\begin{array}{r}4.8 \\
15.4 \\
12.1 \\
10.1 \\
3.3\end{array}$ & $\begin{array}{l}1.2 \\
3.0 \\
2.2 \\
1.2 \\
1.0\end{array}$ & $\begin{array}{r}4.36 \\
14.74 \\
11.36 \\
9.41 \\
2.68\end{array}$ & $\begin{array}{r}4.6 \\
15.8 \\
12.3 \\
10.2 \\
2.9\end{array}$ \\
\hline $\begin{array}{l}1 \\
1 \\
2 \\
3 \\
4 \\
5\end{array}$ & $\begin{array}{l}9 \\
9 \\
9 \\
9 \\
9\end{array}$ & $\begin{array}{l}.46 \\
.67 \\
.72 \\
.72 \\
.63\end{array}$ & $\begin{array}{l}92.4 \\
93.0 \\
92.6 \\
93.4 \\
93.4\end{array}$ & $\begin{array}{l}93.7 \\
94.4 \\
95.6 \\
95.2 \\
95.7\end{array}$ & $\begin{array}{r}7.5 \\
17.4 \\
24.4 \\
10.8 \\
19.6\end{array}$ & $\begin{array}{l}1.3 \\
1.4 \\
3.0 \\
1.8 \\
2.3\end{array}$ & $\begin{array}{r}7.04 \\
16.73 \\
23.68 \\
10.08 \\
18.97\end{array}$ & $\begin{array}{r}7.7 \\
18.1 \\
25.8 \\
10.9 \\
20.4\end{array}$ \\
\hline $\begin{array}{l}1 \\
1 \\
2 \\
3 \\
4 \\
5\end{array}$ & $\begin{array}{l}12 \\
12 \\
12 \\
12 \\
12\end{array}$ & $\begin{array}{l}.44 \\
.63 \\
.74 \\
.71 \\
.59\end{array}$ & $\begin{array}{l}94.0 \\
92.4 \\
93.1 \\
93.7 \\
93.6\end{array}$ & $\begin{array}{l}95.5 \\
97.5 \\
96.2 \\
96.1 \\
96.1\end{array}$ & $\begin{array}{l}23.7 \\
39.6 \\
17.6 \\
13.2 \\
11.7\end{array}$ & $\begin{array}{l}1.5 \\
5.1 \\
3.1 \\
2.4 \\
2.5\end{array}$ & $\begin{array}{l}23.26 \\
38.97 \\
16.86 \\
12.49 \\
11.11\end{array}$ & $\begin{array}{l}24.9 \\
42.5 \\
18.3 \\
13.4 \\
11.9\end{array}$ \\
\hline $\begin{array}{l}1 \\
1 \\
2 \\
3 \\
4 \\
5\end{array}$ & $\begin{array}{l}15 \\
15 \\
15 \\
15 \\
15\end{array}$ & $\begin{array}{l}.47 \\
.65 \\
.76 \\
.71 \\
.66\end{array}$ & $\begin{array}{l}93.6 \\
93.1 \\
93.1 \\
93.6 \\
93.1\end{array}$ & $\begin{array}{l}96.1 \\
95.8 \\
94.9 \\
96.0 \\
95.5\end{array}$ & $\begin{array}{l}51.1 \\
28.0 \\
26.6 \\
39.6 \\
31.4\end{array}$ & $\begin{array}{l}2.5 \\
2.7 \\
1.8 \\
2.4 \\
2.4\end{array}$ & $\begin{array}{l}50.63 \\
27.35 \\
25.84 \\
38.89 \\
30.74\end{array}$ & $\begin{array}{l}54.4 \\
29.6 \\
28.0 \\
41.9 \\
33.3\end{array}$ \\
\hline $\begin{array}{l}1 \\
2 \\
3 \\
4 \\
5\end{array}$ & $\begin{array}{l}18 \\
18 \\
18 \\
18 \\
18\end{array}$ & $\begin{array}{l}.45 \\
.66 \\
.77 \\
.71 \\
.67\end{array}$ & $\begin{array}{l}94.6 \\
92.9 \\
92.9 \\
92.4 \\
94.0\end{array}$ & $\begin{array}{l}96.8 \\
95.3 \\
95.3 \\
96.8 \\
98.3\end{array}$ & $\begin{array}{l}29.6 \\
53.7 \\
67.7 \\
49.5 \\
63.1\end{array}$ & $\begin{array}{l}2.2 \\
2.4 \\
2.4 \\
4.4 \\
4.3\end{array}$ & $\begin{array}{l}29.15 \\
53.04 \\
66.93 \\
48.79 \\
62.43\end{array}$ & $\begin{array}{l}31.0 \\
57.5 \\
72.6 \\
53.2 \\
66.9\end{array}$ \\
\hline
\end{tabular}


TABLE 5.-Effect of exposure to various humidities on the physical properties of the sacks

\begin{tabular}{|c|c|c|c|c|c|c|c|c|c|}
\hline \multirow{2}{*}{ Relative humidity and time } & \multirow{2}{*}{$\begin{array}{l}\text { Sack } \\
\text { No. }\end{array}$} & \multirow{2}{*}{ Length } & \multirow{2}{*}{ Width } & \multicolumn{2}{|c|}{$\begin{array}{l}\text { Threads } \\
\text { per inch }\end{array}$} & \multicolumn{2}{|c|}{$\begin{array}{l}\text { Breaking } \\
\text { strength (1 by } \\
1 \text { by } 3 \text { inch } \\
\text { grab) }\end{array}$} & \multicolumn{2}{|c|}{ Stretch } \\
\hline & & & & Warp & $\begin{array}{l}\text { Fill- } \\
\text { ing }\end{array}$ & Warp & Filling & Warp & Filling \\
\hline High relative humidity, 1 month. & $\begin{array}{l}1 \\
2 \\
3 \\
4 \\
5\end{array}$ & $\begin{array}{r}\text { Inches } \\
291 / 2 \\
29^{3} \\
30 \\
30^{3} / 4 \\
30^{3} / 4\end{array}$ & \begin{tabular}{r|} 
Inches \\
$171 / 4$ \\
175 \\
$181 / 8$ \\
$18 \frac{8}{16}$ \\
$18 \frac{3}{16}$
\end{tabular} & $\begin{array}{l}39 \\
181 / 2 \\
12 \\
13 \\
171 / 2\end{array}$ & $\begin{array}{l}301 / 2 \\
181 / 2 \\
121 / 2 \\
13 \\
18\end{array}$ & $\begin{array}{r}\text { Pounds } \\
90 \\
158 \\
114 \\
105 \\
142\end{array}$ & $\begin{array}{c}\text { Pounds } \\
104 \\
176 \\
210 \\
142 \\
146\end{array}$ & Per cent & $\begin{array}{c}\text { Per cent } \\
\\
\end{array}$ \\
\hline Low relative humidity, 1 month_ & $\begin{array}{l}1 \\
2 \\
3 \\
4 \\
5\end{array}$ & $\begin{array}{l}291 / 4 \\
293 \\
30^{1} / 8 \\
307 / 8 \\
30^{5} / 8\end{array}$ & $\begin{array}{l}17_{16}^{\frac{1}{16}} \\
17^{3} / 4 \\
18_{16}^{\frac{3}{6}} \\
181 / 8 \\
18_{16}^{\frac{1}{16}}\end{array}$ & \begin{tabular}{l|}
39 \\
18 \\
$121 / 2$ \\
13 \\
$171 / 2$
\end{tabular} & $\begin{array}{l}291 / 2 \\
18 \\
13 \\
13 \\
191 / 2\end{array}$ & $\begin{array}{r}78 \\
149 \\
110 \\
109 \\
127\end{array}$ & $\begin{array}{r}89 \\
159 \\
167 \\
120 \\
131\end{array}$ & & $\begin{array}{l}-. . \\
-\cdots . \\
-\cdots . \\
-\cdots\end{array}$ \\
\hline $\begin{array}{l}\text { A verage relative humidity, } 1 \\
\text { month }\end{array}$ & $\begin{array}{l}1 \\
2 \\
3 \\
4 \\
5\end{array}$ & $\begin{array}{l}29 \frac{9}{16} \\
293 / 4 \\
303 / 8 \\
305 / 8 \\
30^{3} / 4\end{array}$ & $\begin{array}{l}171 / 4 \\
175 / 8 \\
18 \frac{3}{16} \\
18 \\
18^{\frac{3}{16}}\end{array}$ & \begin{tabular}{l|}
39 \\
$181 / 2$ \\
12 \\
13 \\
17
\end{tabular} & \begin{tabular}{l|l|}
30 \\
18 \\
$131 / 2$ \\
13 \\
18
\end{tabular} & $\begin{array}{r}78 \\
137 \\
103 \\
110 \\
141\end{array}$ & $\begin{array}{r}87 \\
147 \\
171 \\
111 \\
133\end{array}$ & & \\
\hline High relative humidity, 2 months & $\begin{array}{l}1 \\
2 \\
3 \\
4 \\
5\end{array}$ & $\begin{array}{l}291 / 8 \\
295 / 8 \\
301 / 8 \\
30^{3} \\
301 / 2\end{array}$ & $\begin{array}{l}171 / 8 \\
171 / 2 \\
181 / 8 \\
18 \\
18\end{array}$ & $\begin{array}{l}39 \\
181 / 2 \\
121 / 2 \\
121 / 2 \\
171 / 2\end{array}$ & $\begin{array}{l}301 / 2 \\
181 / 2 \\
13 \\
14 \\
19\end{array}$ & $\begin{array}{r}91 \\
165 \\
117 \\
125 \\
125\end{array}$ & $\begin{array}{l}104 \\
188 \\
209 \\
126 \\
141\end{array}$ & $\begin{array}{r}16 \\
5 \\
3 \\
2 \\
4\end{array}$ & $\begin{array}{l}8 \\
4 \\
2 \\
4 \\
3\end{array}$ \\
\hline Low relative humidity, 2 months & $\begin{array}{l}1 \\
2 \\
3 \\
4 \\
5\end{array}$ & $\begin{array}{l}291 / 2 \\
30^{1 /} \\
30^{1 / 2} \\
30^{3} \\
30^{3} / 4\end{array}$ & $\begin{array}{l}173 / 8 \\
173 \\
181 / 4 \\
181 / 4 \\
181 / 8\end{array}$ & \begin{tabular}{l|}
39 \\
18 \\
$121 / 2$ \\
13 \\
17
\end{tabular} & $\begin{array}{l}30 \\
18 \\
13 \\
13 \\
18\end{array}$ & $\begin{array}{r}75 \\
136 \\
93 \\
92 \\
121\end{array}$ & $\begin{array}{r}86 \\
145 \\
146 \\
115 \\
106\end{array}$ & $\begin{array}{r}15 \\
5 \\
4 \\
3 \\
4\end{array}$ & $\begin{array}{r}10 \\
4 \\
5 \\
4 \\
3\end{array}$ \\
\hline $\begin{array}{l}\text { A verage relative humidity, } 2 \\
\text { months }\end{array}$ & $\begin{array}{l}1 \\
2 \\
3 \\
4 \\
5\end{array}$ & $\begin{array}{l}291 / 2 \\
29^{3} \\
30^{5} / 8 \\
311 / 8 \\
30^{3} / 4\end{array}$ & $\begin{array}{l}173 / 8 \\
175 / 8 \\
181 / 8 \\
183 / 8 \\
181 / 8\end{array}$ & \begin{tabular}{l|}
39 \\
$181 / 2$ \\
12 \\
$121 / 2$ \\
$171 / 2$
\end{tabular} & $\begin{array}{l}301 / 2 \\
18 \\
13 \\
13 \\
171 / 2\end{array}$ & $\begin{array}{r}81 \\
135 \\
102 \\
96 \\
132\end{array}$ & $\begin{array}{r}94 \\
144 \\
146 \\
116 \\
122\end{array}$ & $\begin{array}{r}19 \\
5 \\
3 \\
3 \\
3\end{array}$ & $\begin{array}{r}10 \\
4 \\
4 \\
4 \\
5\end{array}$ \\
\hline High relative humidity, 4 months & $\begin{array}{l}1 \\
2 \\
3 \\
4 \\
5 \\
5\end{array}$ & $\begin{array}{l}291 / 4 \\
295 / 8 \\
303 / 8 \\
30^{5} / 8 \\
301 / 2\end{array}$ & $\begin{array}{l}171 / 8 \\
171 / 2 \\
181 / 8 \\
181 / 8 \\
18\end{array}$ & $\begin{array}{l}39 \\
181 / 2 \\
12 \\
121 / 2 \\
171 / 2\end{array}$ & $\begin{array}{l}301 / 2 \\
19 \\
13 \\
13 \\
18\end{array}$ & $\begin{array}{r}88 \\
156 \\
124 \\
101 \\
134\end{array}$ & $\begin{array}{l}108 \\
175 \\
210 \\
142 \\
152\end{array}$ & \begin{tabular}{r|}
17 \\
5 \\
2 \\
3 \\
2
\end{tabular} & $\begin{array}{r}11 \\
3 \\
4 \\
3 \\
5\end{array}$ \\
\hline Low relative humidity, 4 months. & $\begin{array}{l}1 \\
2 \\
3 \\
4 \\
5\end{array}$ & $\begin{array}{l}293 \\
295 / \\
30^{3} / 2 \\
30^{3} \\
30^{3} / 4\end{array}$ & $\begin{array}{l}171 / 2 \\
173 \\
181 / 8 \\
181 / 4 \\
181 / 4\end{array}$ & $\begin{array}{l}39 \\
18 \\
121 / 2 \\
13 \\
17\end{array}$ & $\begin{array}{l}30 \\
18 \\
13 \\
131 / 2 \\
201 / 2\end{array}$ & $\begin{array}{r}80 \\
134 \\
108 \\
90 \\
120\end{array}$ & $\begin{array}{l}100 \\
164 \\
172 \\
119 \\
142\end{array}$ & $\begin{array}{r}13 \\
3 \\
2 \\
2 \\
2\end{array}$ & $\begin{array}{l}9 \\
3 \\
3 \\
3 \\
2\end{array}$ \\
\hline $\begin{array}{l}\text { A verage relative humidity, } 4 \\
\text { months }\end{array}$ & $\begin{array}{l}1 \\
2 \\
3 \\
4 \\
5\end{array}$ & $\begin{array}{l}295 / 8 \\
301 / 8 \\
301 / 4 \\
311 / 8 \\
307 / 8\end{array}$ & $\begin{array}{l}171 / 2 \\
173 \\
181 / 4 \\
183 / 8 \\
181 / 8\end{array}$ & $\begin{array}{l}39 \\
18 \\
12 \\
121 / 2 \\
171 / 2\end{array}$ & $\begin{array}{l}301^{1 / 2} \\
18 \\
13 \\
131 / 2 \\
201 / 2\end{array}$ & $\begin{array}{r}86 \\
154 \\
114 \\
94 \\
137\end{array}$ & $\begin{array}{r}95 \\
167 \\
192 \\
138 \\
122\end{array}$ & $\begin{array}{r}20 \\
6 \\
2 \\
3 \\
2\end{array}$ & $\begin{array}{l}9 \\
4 \\
4 \\
3 \\
4\end{array}$ \\
\hline Highrelative humidity, 6 months. & $\begin{array}{l}1 \\
2 \\
3 \\
4 \\
5\end{array}$ & $\begin{array}{l}293 / 8 \\
29^{3} / 4 \\
30^{2} \\
30^{7} / 8 \\
30^{3} / 8\end{array}$ & $\begin{array}{l}17 \\
175 / 8 \\
181 / 8 \\
18 \\
181 / 8\end{array}$ & $\begin{array}{l}40 \\
18 \\
12 \\
13 \\
171 / 2\end{array}$ & $\begin{array}{l}30^{1 / 2} \\
18^{1 / 2} \\
13 \\
13 \\
20\end{array}$ & $\begin{array}{r}85 \\
143 \\
106 \\
95 \\
128\end{array}$ & $\begin{array}{l}102 \\
182 \\
218 \\
119 \\
147\end{array}$ & $\begin{array}{r}17 \\
5 \\
2 \\
2 \\
1\end{array}$ & $\begin{array}{r}12 \\
3 \\
2 \\
3 \\
4\end{array}$ \\
\hline Low relative humidity, 6 months. & $\begin{array}{l}1 \\
2 \\
3 \\
4 \\
5\end{array}$ & $\begin{array}{l}291 / 2 \\
293 \\
30^{3} / 8 \\
311 / 8 \\
30^{5} / 8\end{array}$ & $\begin{array}{l}173 / 8 \\
17^{3} \\
181 / 4 \\
181 / 8 \\
181 / 8\end{array}$ & $\begin{array}{l}40 \\
181 / 2 \\
12 \\
13 \\
171 / 2\end{array}$ & $\begin{array}{l}291 / 2 \\
19 \\
13 \\
13 \\
181 / 2\end{array}$ & $\begin{array}{r}80 \\
139 \\
107 \\
107 \\
134\end{array}$ & $\begin{array}{r}89 \\
162 \\
191 \\
118 \\
118\end{array}$ & $\begin{array}{r}12 \\
5 \\
3 \\
3 \\
2\end{array}$ & $\begin{array}{l}9 \\
4 \\
4 \\
4 \\
4\end{array}$ \\
\hline $\begin{array}{l}\text { Average relative humidity, } 6 \\
\text { months }\end{array}$ & $\begin{array}{l}1 \\
2 \\
3 \\
4 \\
5\end{array}$ & $\begin{array}{l}295 / 8 \\
293 / 4 \\
30^{3} / 8 \\
311^{1 / 4} \\
305 / 8\end{array}$ & $\begin{array}{l}173 / 8 \\
173 / 4 \\
181 / 4 \\
181 / 4 \\
181 / 8\end{array}$ & $\begin{array}{l}39 \\
181 / 2 \\
12^{1 / 2} \\
12^{1} / 2 \\
17\end{array}$ & $\begin{array}{l}30 \\
18 \\
121 / 2 \\
13 \\
20^{1} / 2\end{array}$ & $\begin{array}{r}82 \\
149 \\
112 \\
102 \\
123\end{array}$ & $\begin{array}{r}92 \\
158 \\
177 \\
120 \\
144\end{array}$ & $\begin{array}{r}14 \\
2 \\
2 \\
2 \\
3\end{array}$ & $\begin{array}{l}7 \\
2 \\
2 \\
3 \\
3\end{array}$ \\
\hline
\end{tabular}


$\mathrm{T}_{\triangle \mathrm{BLE}}$ 6.-Per cent loss of natural oil in the service test

\begin{tabular}{|c|c|c|c|}
\hline Sack No. & Original & $\begin{array}{l}\text { Service } \\
\text { (fifth } \\
\text { trip) }\end{array}$ & $\begin{array}{l}\text { Hot } \\
\text { cement } \\
\text { (fifth } \\
\text { filling) }\end{array}$ \\
\hline $\begin{array}{l}1 \\
2 \\
3 \\
4 \\
5\end{array}$ & $\begin{array}{c}\text { Per cent } \\
1.65 \\
\text { 3. } 29 \\
3.58 \\
\text { 3. } 23 \\
\text { 4. } 99\end{array}$ & $\begin{array}{c}\text { Per cent } \\
1.55 \\
.73 \\
1.21 \\
1.09 \\
1.87\end{array}$ & $\begin{array}{r}\text { Per cent } \\
1.45 \\
.84 \\
.99 \\
1.00 \\
2.64\end{array}$ \\
\hline
\end{tabular}

TABLE 7.-Effect of time and temperature on sack material

\begin{tabular}{|c|c|c|c|c|c|c|c|c|c|}
\hline \multirow{2}{*}{ Sock No. } & \multirow{2}{*}{$\begin{array}{l}\text { Number } \\
\text { of hours }\end{array}$} & \multicolumn{8}{|c|}{ Average breaking strength at- } \\
\hline & & $200^{\circ} \mathrm{F}$. & $215^{\circ} \mathrm{F}$ & $230^{\circ} \mathrm{F}$. & $245^{\circ} \mathrm{F}$. & $260^{\circ} \mathrm{F}$. & $275^{\circ} \mathrm{F}$ & $290^{\circ} \mathrm{F}$. & $305^{\circ} \mathrm{F}$. \\
\hline 1 1.- & $\begin{array}{r}1 \\
2 \\
4 \\
7 \\
10 \\
15 \\
20 \\
25 \\
30 \\
35 \\
40 \\
45\end{array}$ & $\begin{array}{r}\text { Pounds } \\
76 \\
67 \\
81 \\
84 \\
81 \\
87 \\
82 \\
82 \\
76 \\
72 \\
80 \\
88\end{array}$ & $\begin{array}{r}\text { Pounds } \\
82 \\
78 \\
78 \\
84 \\
89 \\
88 \\
88 \\
84 \\
88 \\
89 \\
84 \\
80\end{array}$ & $\begin{array}{r}\text { Pounds } \\
84 \\
70 \\
72 \\
82 \\
84 \\
86 \\
81 \\
84 \\
80 \\
92 \\
92 \\
88\end{array}$ & $\begin{array}{r}\text { Pounds } \\
72 \\
74 \\
63 \\
84 \\
83 \\
86 \\
69 \\
80 \\
93 \\
88 \\
91 \\
88\end{array}$ & $\begin{array}{r}\text { Pounds } \\
78 \\
82 \\
79 \\
83 \\
81 \\
87 \\
80 \\
79 \\
74 \\
76 \\
80 \\
85\end{array}$ & $\begin{array}{r}\text { Pounds } \\
85 \\
79 \\
86 \\
86 \\
77 \\
89 \\
81 \\
90 \\
88 \\
99 \\
85 \\
87\end{array}$ & $\begin{array}{r}\text { Pounds } \\
80 \\
82 \\
83 \\
85 \\
80 \\
83 \\
76 \\
81 \\
89 \\
89 \\
76 \\
81\end{array}$ & $\begin{array}{r}\text { Pounds } \\
79 \\
80 \\
72 \\
87 \\
80 \\
80 \\
69 \\
79 \\
76 \\
90 \\
77 \\
82\end{array}$ \\
\hline 2 & $\begin{array}{r}1 \\
2 \\
4 \\
7 \\
10 \\
15 \\
20 \\
25 \\
30 \\
35 \\
40 \\
45\end{array}$ & $\begin{array}{l}158 \\
149 \\
147 \\
124 \\
129 \\
170 \\
136 \\
158 \\
133 \\
138 \\
139 \\
153\end{array}$ & $\begin{array}{l}134 \\
137 \\
138 \\
162 \\
156 \\
164 \\
149 \\
154 \\
150 \\
161 \\
154 \\
140\end{array}$ & $\begin{array}{l}125 \\
150 \\
122 \\
142 \\
139 \\
156 \\
149 \\
141 \\
135 \\
166 \\
154 \\
153\end{array}$ & $\begin{array}{l}121 \\
123 \\
117 \\
152 \\
127 \\
166 \\
119 \\
138 \\
161 \\
137 \\
153 \\
151\end{array}$ & $\begin{array}{l}139 \\
133 \\
130 \\
137 \\
147 \\
150 \\
144 \\
150 \\
132 \\
126 \\
132 \\
149\end{array}$ & $\begin{array}{l}155 \\
138 \\
151 \\
171 \\
150 \\
137 \\
138 \\
157 \\
155 \\
138 \\
137 \\
143\end{array}$ & $\begin{array}{l}124 \\
132 \\
136 \\
139 \\
129 \\
134 \\
129 \\
123 \\
144 \\
134 \\
166 \\
130\end{array}$ & $\begin{array}{l}143 \\
145 \\
117 \\
139 \\
123 \\
130 \\
118 \\
118 \\
140 \\
136 \\
145 \\
109\end{array}$ \\
\hline & $\begin{array}{r}1 \\
2 \\
4 \\
7 \\
10 \\
15 \\
20 \\
25 \\
30 \\
35 \\
40 \\
45\end{array}$ & $\begin{array}{l}177 \\
147 \\
164 \\
156 \\
152 \\
176 \\
163 \\
187 \\
157 \\
145 \\
196 \\
174\end{array}$ & $\begin{array}{l}152 \\
152 \\
149 \\
193 \\
160 \\
163 \\
169 \\
164 \\
160 \\
165 \\
168 \\
173\end{array}$ & $\begin{array}{l}142 \\
167 \\
148 \\
156 \\
157 \\
180 \\
162 \\
168 \\
135 \\
178 \\
184 \\
165\end{array}$ & $\begin{array}{l}152 \\
130 \\
126 \\
152 \\
151 \\
187 \\
134 \\
158 \\
161 \\
148 \\
176 \\
155\end{array}$ & $\begin{array}{l}137 \\
147 \\
164 \\
148 \\
172 \\
180 \\
157 \\
168 \\
149 \\
144 \\
167 \\
176\end{array}$ & $\begin{array}{l}171 \\
139 \\
166 \\
157 \\
162 \\
173 \\
145 \\
156 \\
154 \\
159 \\
168 \\
166\end{array}$ & $\begin{array}{l}148 \\
146 \\
163 \\
164 \\
157 \\
147 \\
143 \\
134 \\
157 \\
151 \\
156 \\
155\end{array}$ & $\begin{array}{r}144 \\
180 \\
144 \\
163 \\
157 \\
157 \\
142 \\
126 \\
138 \\
158 \\
112 \\
93\end{array}$ \\
\hline $4-\ldots$ & $\begin{array}{r}1 \\
2 \\
4 \\
7 \\
10 \\
15 \\
20 \\
25 \\
30 \\
35 \\
40 \\
45\end{array}$ & $\begin{array}{r}120 \\
99 \\
107 \\
120 \\
112 \\
129 \\
109 \\
132 \\
120 \\
127 \\
126 \\
135\end{array}$ & $\begin{array}{l}111 \\
110 \\
120 \\
124 \\
120 \\
125 \\
117 \\
129 \\
129 \\
124 \\
115 \\
110\end{array}$ & $\begin{array}{r}109 \\
110 \\
94 \\
124 \\
116 \\
112 \\
109 \\
133 \\
121 \\
129 \\
127 \\
124\end{array}$ & $\begin{array}{r}103 \\
108 \\
92 \\
113 \\
103 \\
132 \\
107 \\
108 \\
131 \\
127 \\
143 \\
125\end{array}$ & $\begin{array}{l}108 \\
103 \\
125 \\
121 \\
127 \\
126 \\
115 \\
110 \\
117 \\
115 \\
114 \\
124\end{array}$ & $\begin{array}{l}116 \\
107 \\
126 \\
127 \\
122 \\
118 \\
108 \\
128 \\
121 \\
114 \\
122 \\
122\end{array}$ & $\begin{array}{r}113 \\
111 \\
118 \\
108 \\
103 \\
121 \\
109 \\
90 \\
109 \\
109 \\
117 \\
105\end{array}$ & $\begin{array}{r}113 \\
120 \\
106 \\
124 \\
100 \\
105 \\
104 \\
97 \\
111 \\
121 \\
90 \\
102\end{array}$ \\
\hline 5 & $\begin{array}{r}1 \\
2 \\
4 \\
7 \\
10 \\
15 \\
20 \\
25 \\
30 \\
35 \\
40 \\
45\end{array}$ & $\begin{array}{l}143 \\
109 \\
130 \\
125 \\
133 \\
141 \\
130 \\
143 \\
140 \\
130 \\
146 \\
164\end{array}$ & $\begin{array}{l}128 \\
126 \\
125 \\
141 \\
141 \\
142 \\
128 \\
129 \\
159 \\
161 \\
134 \\
120\end{array}$ & $\begin{array}{l}129 \\
140 \\
142 \\
149 \\
141 \\
145 \\
132 \\
143 \\
131 \\
150 \\
150 \\
140\end{array}$ & $\begin{array}{l}127 \\
121 \\
105 \\
139 \\
144 \\
143 \\
116 \\
115 \\
140 \\
147 \\
143 \\
141\end{array}$ & $\begin{array}{l}128 \\
126 \\
146 \\
119 \\
135 \\
140 \\
126 \\
134 \\
133 \\
122 \\
136 \\
139\end{array}$ & $\begin{array}{l}141 \\
125 \\
146 \\
129 \\
126 \\
136 \\
124 \\
125 \\
159 \\
155 \\
141 \\
111\end{array}$ & $\begin{array}{l}125 \\
130 \\
136 \\
125 \\
115 \\
135 \\
114 \\
105 \\
127 \\
149 \\
137 \\
127\end{array}$ & $\begin{array}{l}136 \\
132 \\
127 \\
137 \\
120 \\
126 \\
128 \\
109 \\
119 \\
148 \\
119 \\
89\end{array}$ \\
\hline
\end{tabular}




\section{DISCUSSION OF RESULTS}

The ability to withstand rough handling, as shown in this paper, by the test for the resistance to failure from dropping is undoubtedly one of the most important characteristics of a cement container. Besides having a high resistance to drop failure initially, a sack must retain this property throughout a considerable period, and therefore it should be affected as little as possible by service conditions, such as filling with hot cement, handling, transportation in freight cars, or exposures to various humidities. During this investigation sacks were put in service which duplicated the above features and measurements made of the resistance to drop failure at intervals during this service.

Throughout any set of service conditions a sack should be able to afford adequate protection to its contents during damp or rainy weather. A study of this property was made on new sacks only because it was not thought necessary to determine how this resistance to moisture was affected by actual service. This is largely a question of closeness of the weave and absorption qualities of the material.

With these ideas in mind as a basis for selecting the best of the five types tested, there is but little doubt that sack No. 2 should give the longest and most satisfactory service. This sack, as can be seen from Figure 6, has an initial resistance to drop failure greater than any other sack except the osnaburg, and after being in actual service shows the highest resistance of any. Bags Nos. 3 and 4, it will be noted, had very little resistance to drop failure, for after making but two trips these sacks nearly always ruptured on the first drop. These two sacks, therefore, need not be considered further.

The breaking strength and stretch characteristics furnish information which indicates how a sack with high resistance to drop can be constructed, and thus indirectly shows the quality of the sack.

The charts on the results of breaking strength (fig. 6) show that sack No. 2 has a balanced strength condition; that is, the warp and filling strengths are very nearly equal. The results are the highest of all sacks, for although some of the strengths of the filling of sack No. 3 are higher the warp strength is considerably under. It is noted that the per cent stretch of both warp and filling is about equal for sack No. 2. Inasmuch as the filling threads in a sack extend through the length of the sack, they are longer than the warp threads and the actual stretch is greater. Considerable advantage may result by increasing the ability of the warp threads to stretch. This may be accomplished by increasing the crimp of the warp threads by decreasing the warp tension in the loom. In service the load is distributed more equally and causes the warp threads to break less easily. Another improvement is suggested-that a better grade of sewing 
thread be used. Several breaks occurred in the sewing thread during the tests for resistance to drop on sack No. 2.

Figure 5 shows a typical failure during the drop test. The warp threads in the sacks here shown run around the shorter dimension of the sack. The warp threads broke in every case except those cases where the failure was in the sewing thread and not in the fabric.

The results of the effect of hot cement charted in Figure 7 show that the superiority of sack No. 2 over the osnaburg is even greater in this test than in the service test. Its breaking strength and its resistance to drop failure are not at all affected by this treatment, while the qualities of sack No. 1 depreciate markedly. Sack No. 5 deteriorates rapidly at first, but later shows an improvement. This behavior is peculiar, and this anomalous behavior possibly indicates a lack of uniformity in the quality of the sacks themselves rather than an increase in resistance caused by this treatment. Even so, sack No. 5 does not at any time have as great a resistance to drop as sack No. 2, and this fact, together with its lack of uniformity, makes it inferior. Sack No. 1 likewise is definitely inferior in performance to sack No. 2.

The moisture test, results of which are shown graphically in Figure 8 , indicates that the amount of "cake" increases with the length of exposure to dampness. It also shows that all five of the sacks afford equal protection to the cement contained.

\section{SUMMARY}

Five types of cement sacks-a cotton osnaburg and four jute burlap types-were studied to determine their relative merits in regard to their suitability as cement containers.

Various conditions of service were simulated as follows: (a) Repeated shipping between two points about 200 miles apart; $(b)$ filling with hot, freshly ground cement; $(c)$ exposure to various atmospheric conditions; and $(d)$ exposure to excessively damp conditions.

Tests were made of the physical properties in order to determine the effect of these conditions and exposures. These tests included determinations of dimensions, thread count, weight, breaking strength, and resistance to drop. Standard methods of test were followed for all of these except drop, for which no method had been formulated.

A drop test was formulated, as follows: Apparatus ${ }^{1}$ was built which would permit the sack to be dropped from a trap door through a distance of 8 feet to a smooth cement floor. The trapdoor consisted of a double door, upon which the sack was placed lengthwise

\footnotetext{
1 Blue prints may be secured from the Bureau of Standards, Washington, D. C., upon request.
} 
along the line where the doors joined. This arrangement prevented the sack from turning while falling. The test was repeated until the fabrie failed, the number of drops necessary to produce failure indieating the resistanee to drop.

The tests made in this investigation have clearly demonstrated that a cement saek can be made of jute burlap and be not only as good but considerably superior to the more expensive eotton osnaburg tested. It should be borne in mind that only one type of osnaburg sack was used in these tests. Undoubtedly other types may now or eventually be plaeed on the market, whieh may be better than the type used. Of course, jute sacks of poorer quality may prove unsatisfactory, but if the jute sack seleeted is similar to sack No. 2 in this investigation better service, together with considerable saving, price eonditions remaining the same, will result. The tests of this sack made on the original material showed the following charaeteristies: Thread eount, warp 18, filling $181 / 2$; breaking strength (1 by 1 by 3 ineh grab method), warp 153 pounds, filling 167 pounds; weight, 11.8 ounees per square yard; stretch, warp 4 per cent, filling 4 per eent; number of drops necessary before failure, 8 .

Wasinngton, June 26, 1925. 